\title{
Atomistic study of Hydrogen embrittlement of grain boundaries in Nickel: I. Fracture
}

\author{
A. Tehranchi, W. A. Curtin \\ Laboratory for Multiscale Mechanics Modeling, EPFL, CH-1015 Lausanne, Switzerland
}

\begin{abstract}
Hydrogen ingress into a metal is a persistent source of embrittlement. Fracture surfaces are often intergranular, suggesting favorable cleave crack growth along grain boundaries (GBs) as one driver for embrittlement. Here, atomistic simulations are used to investigate the effects of segregated hydrogen on the behavior of cracks along various symmetric tilt grain boundaries in fcc Nickel. An atomistic potential for $\mathrm{Ni}-\mathrm{H}$ is first recalibrated against new quantum level computations of the energy of $\mathrm{H}$ in specific sites within the Ni $\Sigma 5(120)\langle 100\rangle$ GB. The binding energy of $\mathrm{H}$ atoms to various atomic sites in the $\mathrm{Ni} \Sigma 3(111)$ (twin), $\mathrm{Ni} \Sigma 5(120)\langle 100\rangle, \mathrm{Ni} \Sigma 99(557)\langle 110\rangle$, and $\mathrm{Ni} \Sigma 9(221)\langle 110\rangle \mathrm{GBs}$, and to various surfaces created by separating these GBs into two possible fracture surfaces, are computed and used to determine equilibrium $\mathrm{H}$ concentrations at bulk $\mathrm{H}$ concentrations typical of embrittlement in Ni. Mode I fracture behavior is then studied, examining the influence of $\mathrm{H}$ in altering the competition between dislocation emission (crack blunting; "ductile" behavior) and cleavage fracture ("brittle" behavior) for intergranular cracks. Simulation results are compared with theoretical predictions (Griffith theory for cleavage; Rice theory for emission) using the computed surface energies. The deformation behavior at the GBs is, however, generally complex and not as simple as cleavage or emission at a sharp crack tip, which is not unexpected due to the complexity of the GB structures. In cases predicted to emit dislocations from the crack tip, the presence of $\mathrm{H}$ atoms reduces the critical load for emission of the dislocations and no cleavage is found. In the cases predicted to cleave, the presence of $\mathrm{H}$ atoms reduces the cleavage stress intensity and makes cleavage easier, including $\mathrm{Ni} \Sigma 9(221)\langle 110\rangle$ which emits dislocations in the absence of H. Aside from the one unusual $\mathrm{Ni} \Sigma 9(221)\langle 110\rangle$ case, no tendency is found for $\mathrm{H}$ to cause a ductile-to-brittle transformation for cracks along GBs in Ni, either according to theory or simulation for initial equilibrium $\mathrm{H}$ segregation and with

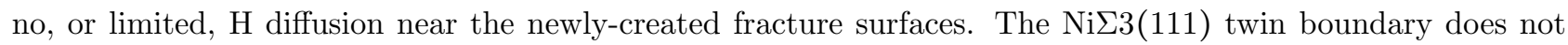
absorb $\mathrm{H}$ at all, suggesting that embrittlement is more difficult in materials with higher fraction of such twin boundaries, as found experimentally. Experimental observations of cleavage-like failure are thus presumably caused by mechanisms involving $\mathrm{H}$ diffusion or dynamic crack growth.
\end{abstract}

Keywords: intergranular fracture, hydrogen embrittlement, directional anisotropy

\section{Introduction}

It is well-established that Hydrogen degrades the mechanical properties of metals such that failure is easier [1 5, but the mechanism(s) driving this embrittlement remain unknown. Hydrogen can interact with (i) dislocations to change the overall plastic flow behavior, (ii) crack tips to change the local deformation (enhancing 5 or suppressing cleavage relative to dislocation emission), (iii) grain boundaries to enhance intergranular failure, (iv) interfaces to enhance interfacial decohesion, and/or (v) other defects such as precipitates, vacancies, and 
solutes. Any combination of these phenomena may occur in a given material, but how these process(es) actually lead to embrittlement (premature failure relative to a Hydrogen-free material) is unclear.

Extensive experimental studies have indirectly revealed phenomena associated with, or affected by, the presence of Hydrogen, at macroscopic, mesoscopic, or atomistic scales. Transmission electron microscopy (TEM) observations show that the separation distance between the dislocations in pile-ups are significantly different in the presence and the absence of hydrogen [6, 7]. There are also experiments that show extensive plastic-like strain localization and slip bands at the vicinity of the crack tips [8, 9. Furthermore, a series of nanoindentation experiments on different ferritic alloys shows that the pop in load denoting the load at which a sudden and significant increase in displacement of the tip of the indenter occurs, reduces by introduction of the cathodically charged hydrogen [10, 11. In tandem with experiments, atomistic simulations are a valuable tool for probing specific mechanisms that occur at the atomic scale, which encompasses most of the above interactions. Thus, there are many studies in the literature where operation of a specific mechanism is studied, and comparisons made between the responses with and without Hydrogen [12 21. Insight from such studies may point to those mechanisms that are most important for embrittlement, as opposed to mechanisms that operate but are tangential or secondary to the failure process.

One feature of Hydrogen embrittlement is often cleavage-like failure, frequently along grain boundaries in polycrystalline metals such as $\mathrm{Ni}$. We thus believe the understanding of embrittlement is likely to be achieved by understanding the behavior of cracks, and at the nanoscale. Cleavage-like failure implies that sharp cracks can propagate without blunting, and this points toward studying crack-tip specific phenomena associated with Hydrogen. We have previously shown that the aggregation of a sufficient amount of $\mathrm{H}$ at a crack tip in bulk $\mathrm{Ni}$ or Fe can prevent dislocation emission and blunting, leaving cleavage as the remaining mechanism to reduce the system energy [19, 20]. Aggregation of $\mathrm{H}$ requires diffusion, and a kinetic model was developed to predict embrittlement as a function of loading rate, $\mathrm{H}$ concentration, and $\mathrm{H}$ diffusion rate, and good agreement was found in application to ferritic (bcc) steels, where $\mathrm{H}$ diffusion is very fast 20 . In polycrystalline metals, segregation of $\mathrm{H}$ to grain boundaries during the $\mathrm{H}$ charging process provides the possibility of embrittlement without active diffusion. That is, if the segregation of $\mathrm{H}$ to a grain boundary can prevent blunting of grain boundary cracks, and permit cleavage along the grain boundary (which is also expected to be easier due to the $\mathrm{H}$ segregation), then the embrittlement may proceed without diffusion. Grain boundary embrittlement by solute species is widely studied, with classic examples such as $\mathrm{S}$ in $\mathrm{Ni}, \mathrm{Bi}$ in $\mathrm{Cu}$, and $\mathrm{Ga}$ in $\mathrm{Al}$. $\mathrm{H}$ embrittlement of grain boundaries has also been studied, but usually using energetic concepts assuming cleavage a priori. The question is then reduced to whether or not $\mathrm{H}$ segregation decreases the work of fracture, and by how much, without consideration of the competing crack tip mode of dislocation emission and blunting. Thus, the aim of the present work is to investigate the effects of $\mathrm{H}$ on modifying the crack tip behavior of cracks along GBs in fcc Ni. We consider GBs containing equilibrium segregation of $\mathrm{H}$ under conditions typical of embrittlement in $\mathrm{Ni}$, and study several different GBs of increasing complexity. To guide understanding, we correlate the results of direct MD simulations to predictions based on the Griffith and Rice theories for cleavage and emission, respectively, and assess whether these theories are useful for predicting or understanding $\mathrm{H}$ embrittlement at GBs.

The remainder of this paper is organized as follows. The Griffith and Rice theories for predicting the critical cleavage and dislocation emission stress intensity factors at the tip of a GB crack in an anisotropic polycrystalline material is reviewed in Section 2. The calibration of a $\mathrm{Ni}-\mathrm{H}$ EAM interatomic potential to 
recent density functional theory (DFT) data for $\mathrm{H}$ in the $\mathrm{Ni} \Sigma 5(120)\langle 100\rangle$ GB and the subsequent energies and segregation of $\mathrm{H}$ to atomic sites in a range of symmetric tilt boundaries in $\mathrm{Ni}$ is presented in Section 3 Section 4 summarizes the details of the simulations of the crack behavior with and without $\mathrm{H}$ segregated to GBs. The results of the simulations are presented and discussed in Sections 5 and 6 , respectively.

\section{Theoretical framework}

In this section, a brief overview of the Griffith and Rice criteria for cleavage and dislocation emission, respectively, and some subtleties in their application to GB problems are discussed. The influence of interstitial hydrogen atoms on the key parameters in each criterion is then also presented.

We consider a symmetric tilt grain boundary with the tilt angle of $\psi$ containing an intergranular crack, as illustrated in Figure 1. The stiffness tensor of the material in the coordinate system $\left\{x_{i}\right\} \quad(i=1,2,3)$ is $C$. The deformation mode at the crack tip is determined by the competition between cleavage and dislocation emission mechanisms. According to Griffith theory, cleavage occurs when the energy release rate at the crack $\operatorname{tip} G=\alpha K_{I \mathrm{c}}^{2}$ reaches the critical value, $G_{c}=\gamma_{\mathrm{i}}=\gamma_{s_{1}}+\gamma_{s_{2}}-\gamma_{G B}$, where $\gamma_{s_{i}}(i=1,2)$, and $\gamma_{G B}$ are the energies of the surfaces made after cleavage and grain boundary energy, respectively. The critical stress intensity factor for cleavage can be determined as

$$
K_{I \mathrm{c}}=\sqrt{\frac{\gamma_{\mathrm{i}}}{\alpha}}
$$

The calculation of $\alpha(\boldsymbol{C}, \psi)$ within anisotropic linear elasticity is well-established, and recently presented in Ref. 22. The surface and interface energies are functions of the crystallographic orientation of the GB and the Hydrogen concentration in the GB.

An important subtlety in using Eq. (1) is that the value for $\gamma_{\mathrm{i}}$ is required but the proper fracture surface for many intergranular crack problems is not well defined. The lowest-energy surface may be kinked (non-flat), which changes the local crack tip stress intensity. The grain boundary structure can also have a long periodic repeat distance, so that the crack could be "trapped" in some local region of the GB where the local energy to extend the crack exceeds the average surface energy. Thus, the critical $K_{I \mathrm{c}}$ computed by the Griffith theory may not be accurate, and could be lower or higher than an assessment based on an assumed fracture surface. The presence of hydrogen makes this problem even more complicated. Hydrogen generally decreases both the surfaces energy and the GB energy, and it is thus not guaranteed that $\gamma_{\mathrm{i}}=\gamma_{s_{1}}+\gamma_{s_{2}}-\gamma_{G B}$ decreases in all cases. We will consider these subtleties below. 


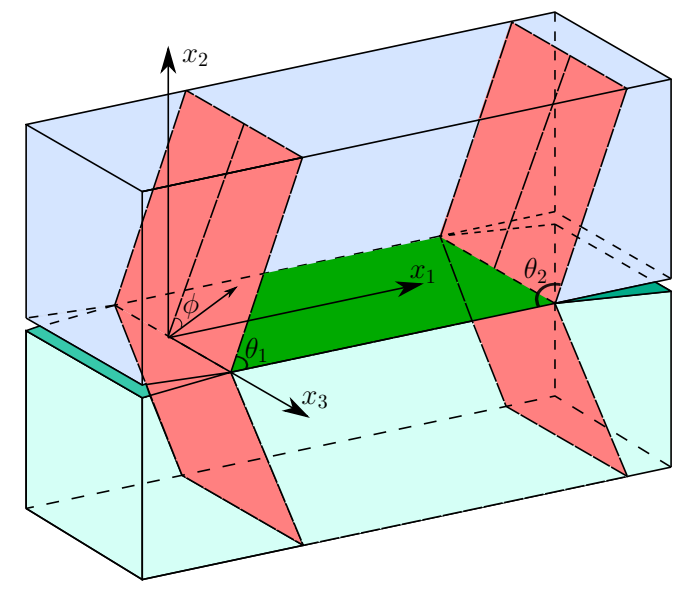

Figure 1: Schematic diagram of a crack along a symmetric tilt boundary, showing the differences in slip plane orientation at the two crack tips.

The emission of a dislocation can occur at the crack tip when the stress intensity $K_{I}$ reaches the critical value $K_{I \mathrm{e}}$ required for a dislocation to be nucleated on a slip system available at the crack tip [23]. For cases where a slip system exists that contains the crack front line, emission of a straight dislocation along the length of the crack can occur, and this is generally the easiest (lowest $K_{I \mathrm{e}}$ ) or most-ductile situation. In this case, the critical $K_{I \mathrm{e}}$ is

$$
K_{I \mathrm{e}}=\sqrt{\beta(\theta, \phi, \mathbf{C}, \psi) \gamma_{\mathrm{usf}}}
$$

where $\theta, \phi$, and $\gamma_{\text {usf }}$ are the angle between the slip and crack planes, the complementary angle of the crack front and the slip direction, and the unstable stacking fault, respectively. Details for calculating $\beta$ within anisotropic elasticity are also given in 22]. The same formulation can be used for the case of the symmetric tilt GBs in cubic metals, which is the case studied here. The unstable stacking fault energy is a function of hydrogen concentration (see, for instance, [21]). However, the slip planes of interest are usually inclined with respect to the grain boundary plane so that $\mathrm{H}$ may exist only very locally at the crack tip along the grain boundary, and it is unclear whether the $\mathrm{H}$ will influence the unstable stacking fault energy that controls the emission. However, in any case, emission is into one of the two grains, and hence the unstable stacking fault energy of the bulk material is probably the relevant quantity. In this work, we use the value of $\gamma_{\text {usf }}$ for pure Ni in all cases, which should provide a lower bound on emission since $\mathrm{H}$ increases the unstable stacking fault energy for the $\mathrm{Ni}-\mathrm{H}$ system [21].

An important feature of intergranular fracture is the directional anisotropy of a crack which is observed in experiments [24, 25]. This feature can be explained by means of Rice theory [23]. Since $K_{I \mathrm{e}}$ depends on $\theta$ and $\phi$, a finite crack with two cracks tips along the same GB has two different values for $\theta$ and $\phi$. One crack tip may show ductile behavior, $K_{I \mathrm{e}}<K_{I \mathrm{c}}$, while the opposite crack tip may show brittle fracture, $K_{I \mathrm{c}}<K_{I \mathrm{e}}$. Here, we define "emission" to refer to cases where emission is theoretically predicted to be favored over cleavage. Likewise, we define "cleavage" to refer to cases where cleavage is theoretically predicted to be favored over emission. Since Ni, and most other fcc metals, do not show intergranular failure in the absence of $\mathrm{H}$, we can safely assume that brittle crack growth in a "cleavage" cases alone is not sufficient for embrittlement. We thus focus mainly on the behavior of the "emission" cases, searching for a ductile-to-brittle transition. 
This is a pre-print of the following article: Tehranchi, A.; Curtin, W. A. Journal Of The Mechanics And Physics Of Solids 2017, 101, 150--165.. The formal publication is available at http://dx.doi.org/10.1016/j.jmps.2017.01.020

\section{Energetics of Hydrogen in Ni grain boundaries and fracture surfaces}

In bulk fcc Ni, H atoms occupy octahedral interstitial sites. The binding sites in and around any particular grain boundary must be determined, and depend strongly on the local atomic structure [26]. For a possible $\mathrm{H}$ site $i$, we define the interaction energy of an $\mathrm{H}$ atom relative to the energy of $\mathrm{H}$ in a bulk octahedral site as

$$
E_{\mathrm{int}, i}=E_{i}^{\mathrm{GB}+\mathrm{H}}-E_{\mathrm{bulk}}^{\mathrm{GB}+\mathrm{H}}
$$

where $E_{i}^{\mathrm{GB}+\mathrm{H}}$ is the energy of a fully-relaxed material containing a grain boundary with an $\mathrm{H}$ atom at site \# $i$

and where $E_{\text {bulk }}^{\mathrm{GB}+\mathrm{H}}$ the energy of the same fully-relaxed material with an $\mathrm{H}$ atom in a bulk interstitial site far from the GB.

\subsection{Validated $N i-H$ interatomic potential}

For the complex crack studies here, atomistic simulations must use semi-empirical interatomic potentials; direct first principles computations are prohibitively expensive. It is thus essential that the interatomic potential function be validated against higher-fidelity calculations. Here, we adapt the embedded atom method (EAM) potential for $\mathrm{Ni}-\mathrm{H}$ introduced by Angelo et al. [27, 28, and previously modified by Song and Curtin [19], to obtain good agreement with recent results obtained using density functional theory (DFT) (Alvaro et al. 29], Di Stefano et al. 30]) for the case of $\mathrm{H}$ in $\mathrm{Ni} \Sigma 5(120)\langle 100\rangle$. Figure 2a shows the positions of the binding sites found in the two different sets of DFT calculations; the corresponding H/GB interaction energies are shown in Table 1. Alvaro et al. 29] show that $\mathrm{H}$ binds to octahedral-like sites at the boundary, with site \#5 in a very open region of the GB being unstable. This result is slightly counter-intuitive because $\mathrm{H}$ atoms have large misfit volume and so generally prefer to occupy sites with more open structure, and surfaces are also quite favorable relative to the bulk. Di Stefano et al. 30. show this site to be stable and with a large interaction energy of $-0.22 \mathrm{eV}$. In spite of the discrepancy at site \#5, the results are comparable for $\mathrm{H}$ at binding sites \#1 and \#2. The EAM potential finds all the octahedral-like sites \#1-\#4 and the site \#5 to be stable and binding. For the existing $\mathrm{Ni}-\mathrm{H}$ EAM potential, however, the interaction energies at all sites are $0.1 \mathrm{eV}$ higher (stronger binding) than the DFT calculations. This discrepancy would lead to large overestimation of the probability of occupation of such sites in the $\mathrm{Ni} \Sigma 5(120)\langle 100\rangle$ as well as errors in the GB and surface energies in the presence of $\mathrm{H}$. The existing Ni-H EAM potential is thus inadequate.

Table 1: Interaction energies for Hydrogen atoms in $\mathrm{Ni}(120) \Sigma 5\langle 100\rangle$ at various sites indicated in Figure 2a Results are shown for computations using DFT [Alvaro et al. [29], Di Stefano et al. [30]], using the original Ni-H EAM potential [19], and using the modified $\mathrm{Ni}-\mathrm{H}$ EAM potential developed in this paper.

\begin{tabular}{ccccc}
\hline \multicolumn{5}{c}{$E_{\text {int }}(\mathrm{eV})$} \\
\hline \# site & DFT [29] & DFT [30] & EAM [19] & Modified EAM (this work) \\
\hline 1 & -0.23 & -0.23 & -0.37 & -0.25 \\
2 & -0.14 & -0.17 & -0.26 & -0.13 \\
3 & -0.09 & not studied & -0.24 & -0.16 \\
4 & +0.04 & not studied & -0.09 & -0.05 \\
5 & not stable & -0.22 & -0.28 & -0.14 \\
6 & not stable & -0.09 & +0.05 & +0.07 \\
\hline
\end{tabular}


We have modified the existing EAM potential by changing some of the parameters that describe the $\mathrm{Ni}-\mathrm{H}$ interactions but while maintaining good overall properties. Table 2 shows the original and revised values for the modified parameters in the potential as well as the relevant bulk properties of $\mathrm{Ni}$ that are used in the analyses provided in this work. With the new potential, the positions of the energetically favorable sites for $\mathrm{H}$ remain unchanged but the binding energies are closer to the DFT calculations, as shown in Table 1 The surface energy of $\mathrm{Ni}(111)$ with $\mathrm{H}$ has also been computed as 31]

$$
\gamma_{\mathrm{s}}=\frac{1}{2 A}\left(E_{\mathrm{s}}\left(N^{\mathrm{Ni}}, N^{\mathrm{H}}\right)-N^{\mathrm{Ni}} E_{\text {coh }}^{N i}-N^{\mathrm{Ni}} E_{\text {bulk }}^{H}\right)
$$

\subsection{Energies of $H$ in grain boundaries and fracture surfaces}

We have used the new $\mathrm{Ni}-\mathrm{H}$ potential to find binding sites for $\mathrm{H}$ and their associated interaction energies in and around the $\mathrm{Ni} \Sigma 3(111)\langle 110\rangle, \mathrm{Ni} \Sigma 9(221)\langle 110\rangle$, and $\mathrm{Ni} \Sigma 99(557)\langle 110\rangle$ grain boundaries. Figure 2 shows the position of the binding sites for $\mathrm{H}$ in each GB. The corresponding interaction energies are shown in Table 3 . It should be noted that the molecular statics simulations are performed using the Large-scale Atomic/Molecular Massively Parallel Simulator (LAMMPS) [32 and atomic configurations are visualized using the Open Visualization Tool (OVITO) 33]. For Ni $\Sigma 3(111)\langle 110\rangle$, the twin boundary, there is only one site for $\mathrm{H}$ and it has a very small interaction energy. For $\mathrm{Ni} \Sigma 9(221)\langle 110\rangle$ and $\mathrm{Ni} \Sigma 99(557)\langle 110\rangle$, the binding sites are concentrated in the areas with more open structure. We have evaluated the $\mathrm{H}-\mathrm{H}$ interactions for $\mathrm{H}$ atoms in two types of sites; in all cases, the $\mathrm{H}-\mathrm{H}$ interactions are repulsive but fairly small (all less than $0.02 \mathrm{eV}$ ). We thus neglect the $\mathrm{H}-\mathrm{H}$ interactions and consider the interaction energy of each site as independent of the $\mathrm{H}$ occupation of any other sites. We followed the same procedure to find the binding sites and energies on and around the free surfaces 
This is a pre-print of the following article: Tehranchi, A.; Curtin, W. A. Journal Of The Mechanics And Physics Of Solids 2017, 101, 150--165.. The formal publication is available at http://dx.doi.org/10.1016/j.jmps.2017.01.020

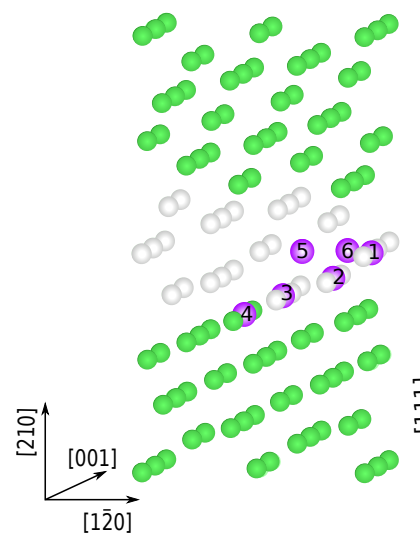

(a) $\mathrm{Ni} \Sigma 5(120)\langle 100\rangle$

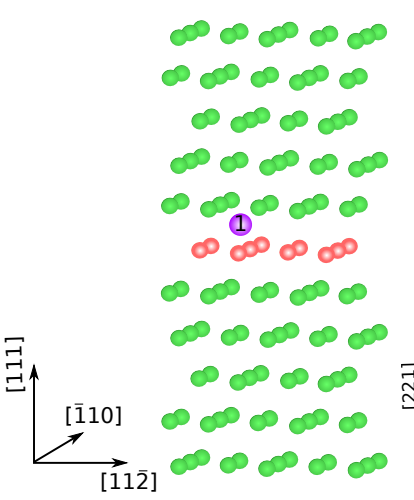

(b) $\mathrm{Ni} \Sigma 3(111)\langle 110\rangle$

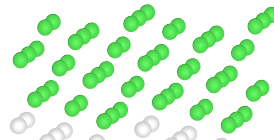

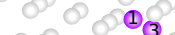

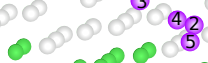

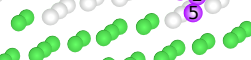
-

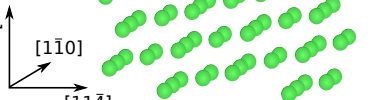

(c) $\operatorname{Ni} \Sigma 9(221)\langle 110\rangle$

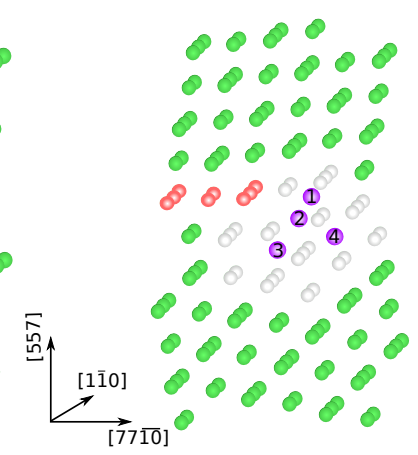

(d) $\mathrm{Ni} \Sigma 99(557)\langle 110\rangle$
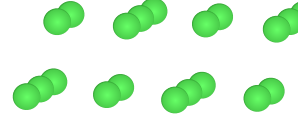

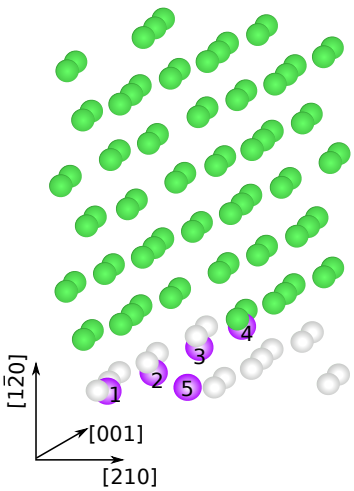

(e) $\mathrm{Ni}(111)$

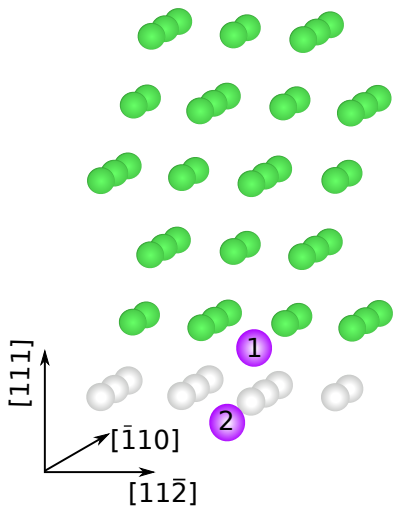

(f) $\mathrm{Ni}(110)$

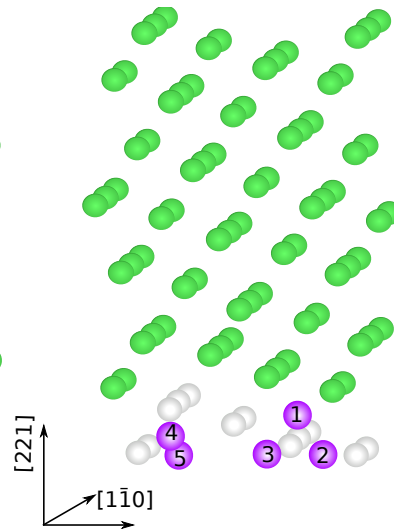

$[11 \overline{4}]$

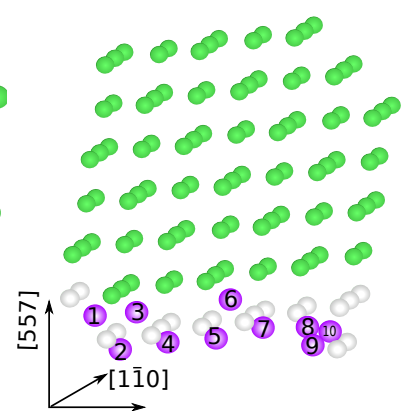

$[\overrightarrow{7} 7 \overline{10}]$

(g) $\mathrm{Ni}(221)$

Figure 2: Structure and Hydrogen binding sites in various symmetric tilt boundaries and free surfaces: (a) Ni $\Sigma 5(120)\langle 100\rangle$; (b)

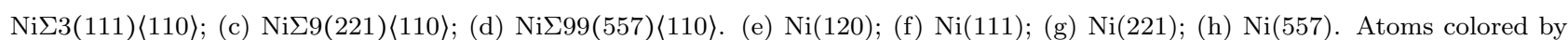
Common Neighbor Analysis (Green: fcc Ni; Red: hcp Ni; White: Ni with less than 12 neighbors; Purple: Hydrogen sites)

that are related to surfaces created by advancement of the intergranular crack along the grain boundary. The position of the binding sites for $\mathrm{Ni}(111), \mathrm{Ni}(120), \mathrm{Ni}(221)$, and $\mathrm{Ni}(557)$ are illustrated in Figure 2 and the interaction energies are displaced in Table 3. The interaction energies of $\mathrm{H}$ atoms with the free surfaces are necessary for calculation of the equilibrium concentration of the $\mathrm{H}$ atoms on the initial crack faces, but do not enter in any other calculations here. It also should be mentioned that the repulsive $\mathrm{H}-\mathrm{H}$ interaction of the adjacent sites at these surfaces is also negligible. 
This is a pre-print of the following article: Tehranchi, A.; Curtin, W. A. Journal Of The Mechanics And Physics Of Solids 2017, 101, 150--165.. The formal publication is available at http://dx.doi.org/10.1016/j.jmps.2017.01.020

Table 3: Interaction energies for Hydrogen atoms at various sites in $\mathrm{Ni} \Sigma 3\langle 110\rangle, \mathrm{Ni} \Sigma 9(221)\langle 110\rangle, \mathrm{Ni} \Sigma 99(557)\langle 110\rangle \mathrm{GBs}$ and $\mathrm{Ni}(111)$, $\mathrm{Ni}(120), \mathrm{Ni}(221), \mathrm{Ni}(557)$ surfaces as predicted by the modified EAM potential.

\begin{tabular}{|c|c|c|c|c|c|c|c|}
\hline \multicolumn{8}{|c|}{$E_{\text {int }}(e V)$} \\
\hline \# site & $\mathrm{Ni} \Sigma 3(111)\langle 110\rangle$ & $\mathrm{Ni} \Sigma 9(221)\langle 110\rangle$ & $\mathrm{Ni} \Sigma 99(557)\langle 110\rangle$ & $\mathrm{Ni}(111)$ & $\mathrm{Ni}(120)$ & $\operatorname{Ni}(221)$ & $\mathrm{Ni}(557)$ \\
\hline 1 & -0.04 & -0.20 & -0.15 & -0.19 & -0.55 & -0.22 & -0.16 \\
\hline 2 & - & -0.16 & -0.20 & -0.47 & -0.55 & -0.51 & -0.56 \\
\hline 3 & - & -0.16 & -0.26 & - & -0.24 & -0.46 & -0.23 \\
\hline 4 & - & -0.20 & -0.21 & - & -0.07 & -0.46 & -0.48 \\
\hline 5 & - & -0.16 & - & - & -0.43 & -0.48 & -0.48 \\
\hline 6 & - & - & - & - & - & - & -0.21 \\
\hline 7 & - & - & - & - & - & - & -0.48 \\
\hline 8 & - & - & - & - & - & - & -0.43 \\
\hline 9 & - & - & - & - & - & - & -0.48 \\
\hline 10 & - & - & - & - & - & - & -0.61 \\
\hline
\end{tabular}

Based on the computed binding energies and small magnitudes of $\mathrm{H}-\mathrm{H}$ interactions, we can construct GB structures with equilibrium segregation of $\mathrm{H}$. We use a simple solution model to compute the concentration $c_{i}$ of $\mathrm{H}$ segregated to sites of type $i$ with binding energy $E_{\mathrm{int}, i}$ as

$$
c_{i}=\frac{c_{0} \exp \left(-\mathrm{E}_{\mathrm{int}, i} / \mathrm{k}_{\mathrm{B}} \mathrm{T}\right)}{1+c_{0} \exp \left(-\mathrm{E}_{\mathrm{int}, i} / \mathrm{k}_{\mathrm{B}} \mathrm{T}\right)}
$$

where $T$ is the temperature, $c_{0}$ is the bulk concentration of $\mathrm{H}$ atoms, corresponding to some imposed $\mathrm{H}$ chemical potential during charging, and $k_{\mathrm{B}}$ is Boltzmann's constant. The simple solution model neglects specific entropy contributions beyond the entropy of mixing. Given a desired concentration $c_{i}$, specific atomic sites of type $i$ are occupied with $\mathrm{H}$ by selecting a random number in the domain $R \in[0,1]$ and occupying the specific site if $R<c_{i}$.

For cleavage fracture, we require the initial grain boundary energy $\gamma_{G B}$ and the final energies of the two new fracture surfaces $\gamma_{s 1}, \gamma_{s 2}$ that are created by the cleavage. These quantities combine to yield $\gamma_{\mathrm{i}}=\gamma_{s_{1}}+\gamma_{s_{2}}-\gamma_{G B}$ that enters the Griffith theory for cleavage. The GB energies without H are easily computed. In the presence of $\mathrm{H}$, we can also compute the total energy knowing the $\mathrm{H} / \mathrm{GB}$ interaction energies $E_{\mathrm{int}, i}$ and the equilibrium concentrations $c_{i}$ for all site types $i$ per unit of GB area. The final surface energies $\gamma_{s 1}, \gamma_{s 2}$ depend, however, on the precise fracture surface, which is not defined a priori. Imagining fracture to occur by the separation across a specified planar surface, we can consider several possible planar surfaces and compute the energy $\gamma_{s 1}+\gamma_{s 2}$ for each planar surface. We compute the energy for both rigid separation and following atomistic relaxation of the separated surfaces to examine the effects of such relaxations. We then select the lowest energy among all of these choices as the final surface energy used in the theory. For each GB, the three different choices of planar surface are shown in Figures 35 and the resulting final surface energies are shown in Table 4 , for cases with and without H. Table 4 shows that the variations in $\gamma_{\mathrm{i}}$ versus choice of separation plane are generally not large for the fully relaxed surfaces. The largest differences upon relaxation occur for those surfaces with high unrelaxed energies. More interestingly, the presence of $\mathrm{H}$ does not lead to significant changes in the fracture energy $\gamma_{\mathrm{i}}$, except for the case of the $\mathrm{Ni}(120) \Sigma 5\langle 100\rangle \mathrm{GB}$, which is expected to be brittle even without $\mathrm{H}$. 
This is a pre-print of the following article: Tehranchi, A.; Curtin, W. A. Journal Of The Mechanics And Physics Of Solids 2017, 101, 150--165.. The formal publication is available at http://dx.doi.org/10.1016/j.jmps.2017.01.020

The above calculations ignore the possibility of $\mathrm{H}$ diffusion near the crack tip during the (possible) crack opening. These results are thus the "fast" limit considered by Rice 34, in contrast to the "slow" limit where the final fracture surfaces are considered to have full equilibrium H concentrations of the surfaces. The "slow" limit is achieved by $\mathrm{H}$ diffusion, and at very low bulk $\mathrm{H}$ concentrations would require diffusion over significant distances continuously during crack growth. Here, we consider another more realistic limit wherein all $\mathrm{H}$ that is near the fracture surface, due to having been segregated to the GB prior to fracture, can diffuse via a few atomic hops to some lower-energy position on the fracture surface. Such diffusion could be envisioned to occur right at the crack tip during the opening process. To examine this "local diffusion" case, we have started from the relaxed separated fracture surfaces and then moved all subsurface $\mathrm{H}$ atoms to nearby low-energy sites on the fracture surface. These atomic configurations are shown in Figure 3 5. The entire system is then relaxed to obtain new energies $\gamma_{s 1}, \gamma_{s 2}$ and hence new fracture energies $\gamma_{\mathrm{i}}$, as shown in Table 4 . We find very limited changes in the fracture energy due to this local diffusion, especially for the lowest fracture-energy cases that control the fracture behavior.

Figs. 35 shows the structure of the grain boundaries of interest in the presence and absence of $\mathrm{H}$ atoms. The relaxed and unrelaxed states of the separated grain boundaries for different cutting planes are given in these Figures as well. It should be noted that since the interaction energy of the $\mathrm{H}$ atoms with the twin boundary is extremely low, the amount of $\mathrm{H}$ atoms along the boundary is low such that the separation energy in the $\mathrm{H}$-charged cases is identical to that of the H-free case. Thus, local diffusion effects are also insignificant.

Table 4: Unrelaxed and fully relaxed fracture energies $\left(\mathrm{mJ} / \mathrm{m}^{2}\right)$ for the separation of GBs along different possible fracture planes. The minimum separation energy for each GB is highlighted.

\begin{tabular}{|c|c|c|c|c|c|c|c|c|c|}
\hline & & \multicolumn{3}{|c|}{$c_{0}=0$} & \multicolumn{3}{|c|}{$c_{0}=0.001$} & \multicolumn{2}{|c|}{$c_{0}=0.001$ (local diffusion) } \\
\hline & & unrelaxed & relaxed & reduction $(\%)$ & unrelaxed & relaxed & reduction $(\%)$ & relaxed & reduction $(\%)$ \\
\hline \multirow{3}{*}{$\mathrm{Ni} \Sigma 9(221)\langle 110\rangle$} & $P_{1}$ & 3625 & 3438 & 5 & 3562 & 3377 & 4.9 & 3377 & 0 \\
\hline & $P_{3}$ & 4753 & 3427 & 27 & 4745 & 3437 & 27 & 3370 & 1.9 \\
\hline & $P_{1}$ & 4378 & 3718 & 15.1 & 4853 & 3801 & 21 & 3787 & 0.5 \\
\hline \multirow[t]{3}{*}{$\mathrm{Ni} \Sigma 99(557)\langle 110\rangle$} & $P_{2}$ & 4162 & 3273 & 21.3 & 4170 & 3191 & 23 & 3162 & 0.9 \\
\hline & $P_{3}$ & 4475 & 3756 & 16 & 4484 & 3541 & 21 & 3515 & 0.7 \\
\hline & $P_{1}$ & 4145 & 3716 & 10.3 & 3020 & 2135 & 29.3 & 2135 & 0.0 \\
\hline \multirow[t]{2}{*}{$\mathrm{Ni} \Sigma 5(120)\langle 100\rangle$} & $P_{2}$ & 4145 & 3716 & 10.3 & 3122 & 2145 & 31.3 & 2257 & -5.2 \\
\hline & $P_{3}$ & 5176 & 3732 & 27.9 & 4638 & 2909 & 37.3 & 2208 & 24.9 \\
\hline
\end{tabular}


This is a pre-print of the following article: Tehranchi, A.; Curtin, W. A. Journal Of The Mechanics And Physics Of Solids 2017, 101, 150--165.. The formal publication is available at http://dx.doi.org/10.1016/j.jmps.2017.01.020

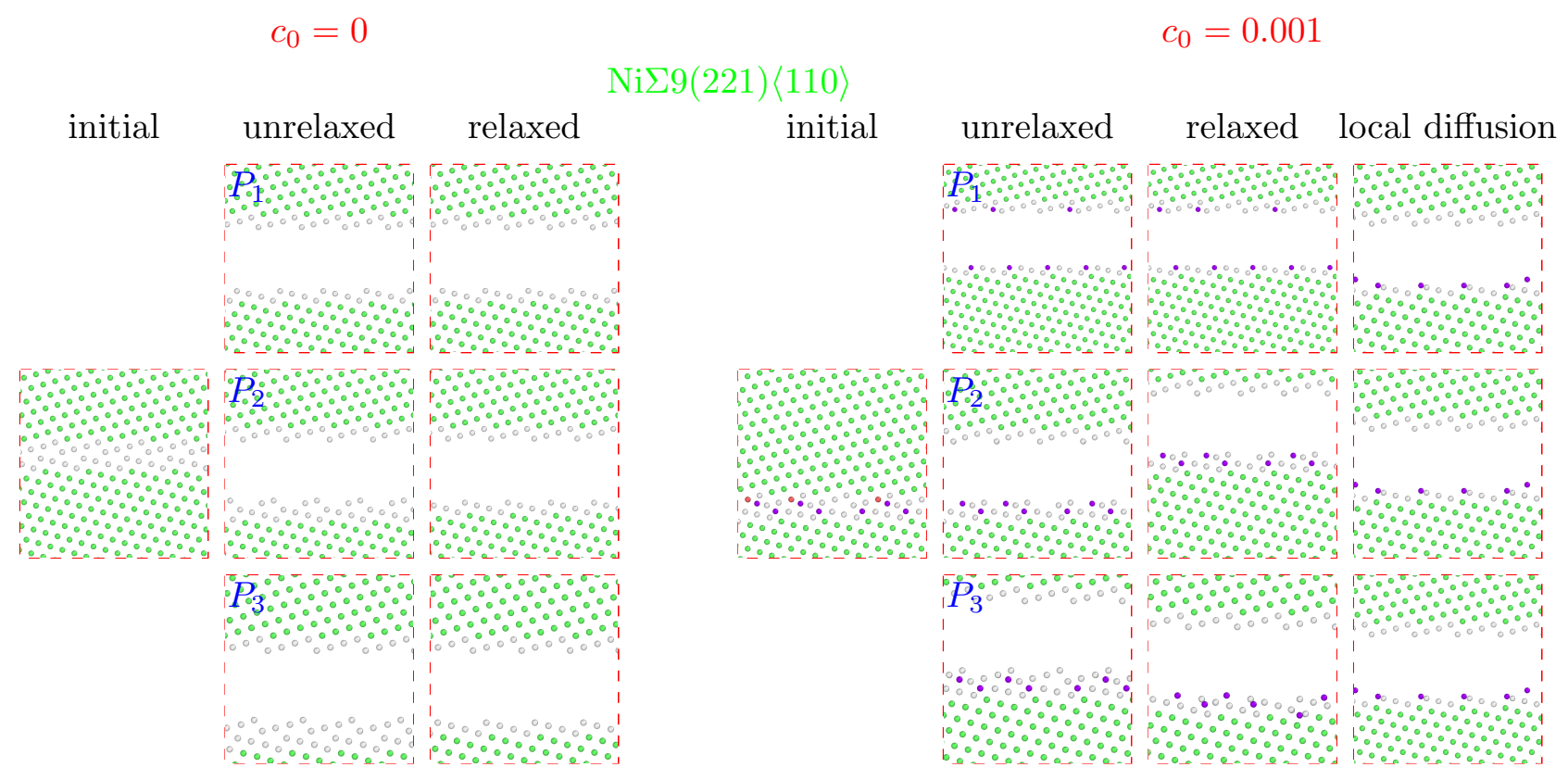

Figure 3: Atomic configurations of $\mathrm{Ni} \Sigma 9(221)\langle 110\rangle$ with and without hydrogen. For each grain boundary, the relaxed and unrelaxed configuration of the separated grains through three different cutting planes are given. Atoms colored by Common Neighbor Analysis (Green: fcc Ni; Red: hcp Ni; White: Ni with less than 12 neighbors; Purple: H)

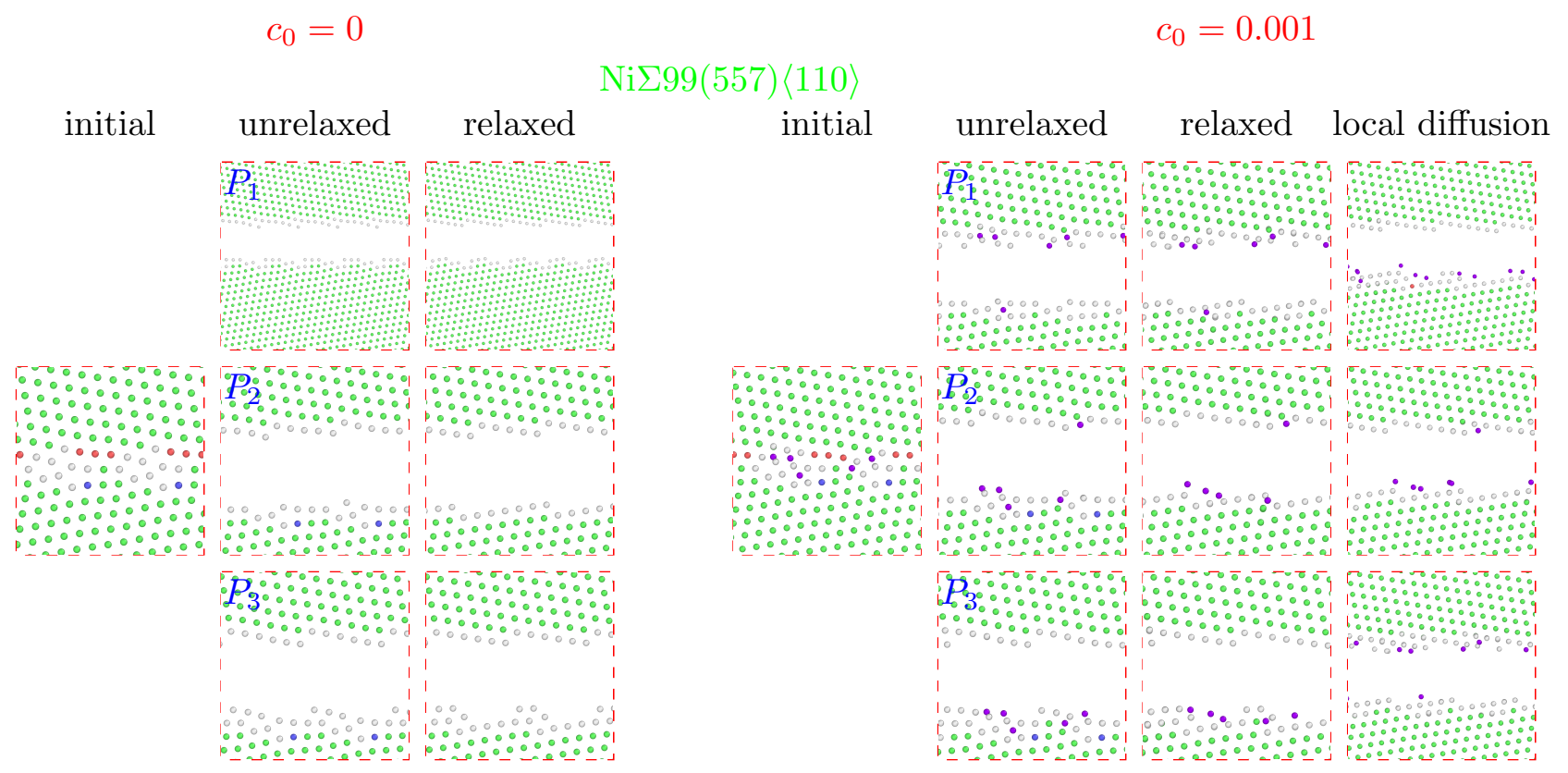

Figure 4: Atomic configurations of $\mathrm{Ni \Sigma}$ 99(557) $\langle 110\rangle$ with and without hydrogen. For each grain boundary, the relaxed and unrelaxed configuration of the separated grains through three different cutting planes are given. Atoms colored by Common Neighbor Analysis (Green: fcc Ni; Red: hcp Ni; White: Ni with less than 12 neighbors; Purple: H) 
This is a pre-print of the following article: Tehranchi, A.; Curtin, W. A. Journal Of The Mechanics And Physics Of Solids 2017, 101, 150--165.. The formal publication is available at http://dx.doi.org/10.1016/j.jmps.2017.01.020

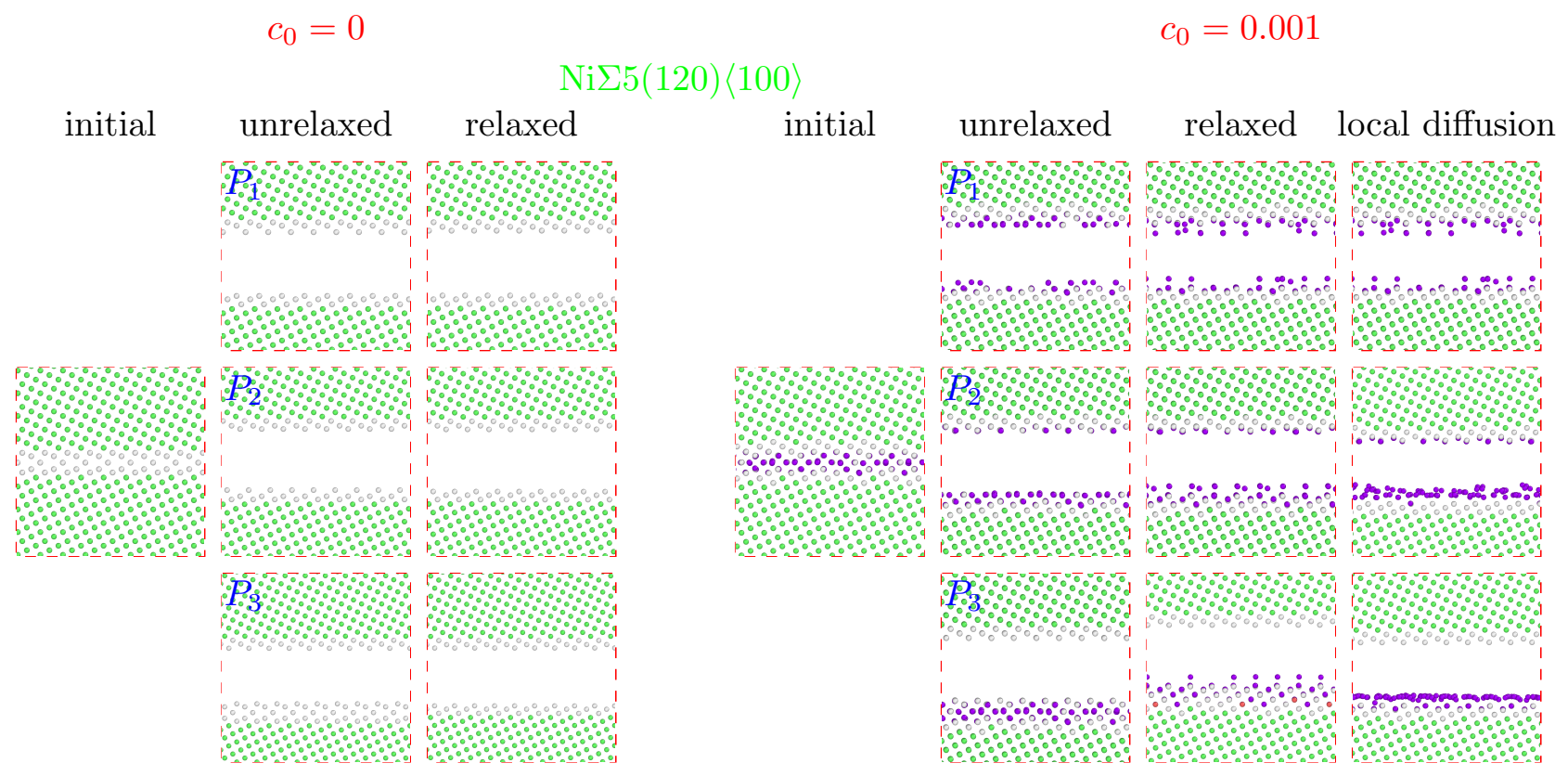

Figure 5: Atomic configurations of $\mathrm{Ni} \Sigma 5(120)\langle 100\rangle$ with and without hydrogen. For each grain boundary, the relaxed and unrelaxed configuration of the separated grains through three different cutting planes are given. Atoms colored by Common Neighbor Analysis (Green: fcc Ni; Red: hcp Ni; White: Ni with less than 12 neighbors; Purple: H)

\section{Fracture simulations}

With the above baseline of GB and fracture energies with and without $H$, we now examine the explicit simulation of crack-tip processes in molecular statics. We construct simulation cells of size $l_{1} \in[420 \AA, 490 \AA]$, $l_{2} \in[400 \AA, 500 \AA]$ and $l_{3} \in[17 \AA, 20 \AA]$ containing a GB $(\mathrm{Ni} \Sigma 9(221)\langle 110\rangle, \mathrm{Ni} \Sigma 99(557)\langle 110\rangle, \mathrm{Ni} \Sigma 5(120)\langle 100\rangle$ or $\mathrm{Ni} \Sigma 3(111)\langle 110\rangle)$ along the central plane $x_{2}=0$. For all grain boundaries except the twin boundary, we insert a crack in the $x_{1} x_{3}$ plane of the grain boundary with crack front along [11̄0] by removing three atomic layers. For the twin, using a blunted crack leads to unphysical results and so a sharp crack is inserted by artificially eliminating atomic interactions across the crack faces. For this crack geometry, all cases except Ni $\Sigma 5(120)\langle 100\rangle$ have a slip plane that allows for the emission of straight dislocations from the crack tip. For the cases with $\mathrm{H}$ atoms, we saturate the binding sites of the crack faces at the equilibrium concentrations $c_{i}$ according to Eq. (5). To study the directional anisotropy of the intergranular cracks, we consider crack growth in both emission and cleavage directions, and both directions for the Ni $\Sigma 5(120)\langle 100\rangle$ GB. The simulation cell is then subjected to incremental $K$-field loading by displacing all atoms within $10 \AA ̊$ of the outer cell boundary according to the asymptotic continuum solution for a semi-infinite crack in an anisotropic linear elastic symmetric bicrystal. After each increment of loading, the boundary of the specimen is held fixed and the remaining atoms in the simulation cell are relaxed to the minimum total energy of the system using the conjugated gradient method [35. The relevant geometric parameters describing the slip in the grains are tabulated in Table 5. 
This is a pre-print of the following article: Tehranchi, A.; Curtin, W. A. Journal Of The Mechanics And Physics Of Solids 2017, 101, 150--165.. The formal publication is available at http://dx.doi.org/10.1016/j.jmps.2017.01.020

Table 5: Geometrical parameters describing slip orientations in grains adjacent to each grain boundary.

\begin{tabular}{cccc}
\hline GB & case & $\phi\left(^{\circ}\right)$ & $\theta\left(^{\circ}\right)$ \\
\hline \multirow{2}{*}{$\mathrm{Ni} \Sigma 3(111)\langle 110\rangle$} & cleavage & 60 & 109.5 \\
& emission & 0 & 70.5 \\
\cline { 2 - 4 } $\mathrm{Ni} \Sigma 9(221)\langle 110\rangle$ & cleavage & 60 & 125 \\
& emission & 0 & 55 \\
\cline { 2 - 4 } $\mathrm{Ni} \Sigma 99(557)\langle 110\rangle$ & cleavage & 60 & 103 \\
& emission & 0 & 77 \\
\cline { 2 - 4 } $\mathrm{Ni} \Sigma 5(120)\langle 100\rangle$ & cleavage & 45 & N.A. \\
& emission & 45 & N.A. \\
\hline
\end{tabular}

Note that we use $\mathrm{H}$ segregation computed at finite temperature but execute the simulations at zero $\mathrm{T}$. This ensures that there is no diffusion during the simulation, nor any other thermally activated behavior, so that the simulation probes the energetics of fracture with no time dependence. Performing finite-T molecular dynamics would introduce a time scale into the problem, and all results would then depend on the simulation time and/or loading rate. Since MD loading rates are unrealistically high, as compared to experiments, the finite- $\mathrm{T} /$ finite-time results would provide little or no additional insights beyond the $\mathrm{T}=0 K$ simulations. It is then clear that, for comparison with theory, the relevant interface energy in the Griffith theory is the minimum value of $\gamma_{\mathrm{i}}$ without local diffusion as shown in Table 4.

\section{Results}

Table 6 summarizes the results of the theoretical predictions of the Griffith and Rice theories as well as the simulation results. The values for the theoretical cleavage loads are obtained by inserting the corresponding information in Table 4 into Eq. (1) while the theoretical emission loads are calculated by incorporating the information in Tables 2 and 5 into Eq. (2). 
This is a pre-print of the following article: Tehranchi, A.; Curtin, W. A. Journal Of The Mechanics And Physics Of Solids 2017, 101, 150--165.. The formal publication is available at http://dx.doi.org/10.1016/j.jmps.2017.01.020

Table 6: Theoretical cleavage and emission stress intensities for all grain boundaries studied here. The predicted behavior (cleavage or emission) is indicated, as dictated by the lower of the two predicted stress intensities as highlighted in bold. The simulation results are tabulated as well.

\begin{tabular}{ccccc}
\hline $\mathrm{Ni} \Sigma 3(111)\langle 110\rangle$ & $c_{0}$ & $\begin{array}{r}\text { Rice theory } \\
(\mathrm{MPa} \sqrt{\mathrm{m}})\end{array}$ & $\begin{array}{c}\text { Griffith theory } \\
(\mathrm{MPa} \sqrt{\mathrm{m}})\end{array}$ & $\begin{array}{c}\text { simulation results } \\
(\mathrm{MPa} \sqrt{\mathrm{m}})\end{array}$ \\
\hline \multirow{3}{*}{$\mathrm{Ni} \Sigma 3(111)\langle 110\rangle$} & 0.0 & $\mathbf{1 . 0 1}$ & 1.03 & 1.20 cleavage \\
& 0.001 & $\mathbf{1 . 0 1}$ & 1.03 & 1.20 cleavage \\
& 0.0 & $\mathbf{0 . 3 8}$ & 1.03 & 0.62 emission \\
& 0.001 & $\mathbf{0 . 3 8}$ & 1.03 & 0.58 emission \\
\cline { 2 - 5 } $\mathrm{Ni} \Sigma 9(221)\langle 110\rangle$ & 0.001 & 1.500 & $\mathbf{0 . 9 8}$ & 1.49 rearrangement \\
& 0.0 & $\mathbf{0 . 6 4}$ & 0.98 & 1.39 cleavage \\
& 0.001 & $\mathbf{0 . 6 4}$ & 0.97 & 0.72 emission \\
\cline { 2 - 5 } $\mathrm{Ni} \Sigma 99(557)\langle 110\rangle$ & 0.0 & 1.41 & $\mathbf{1 . 0 0}$ & 1.52 rearrangement \\
& 0.001 & 1.41 & $\mathbf{0 . 9 2}$ & 1.07 cleavage \\
& 0.0 & $\mathbf{0 . 6 2}$ & 1.00 & 0.63 emission \\
& 0.001 & $\mathbf{0 . 6 2}$ & 0.92 & 0.58 emission \\
\cline { 2 - 5 } & 0.0 & N.A. & 0.92 & 0.91 cleavage \\
& 0.001 & N.A. & 0.75 & 0.65 cleavage \\
$\mathrm{Ni} \Sigma 5(120)\langle 100\rangle$ & 0.0 & N.A. & 0.92 & 1.38 cleavage \\
& 0.001 & N.A. & 0.75 & 1.20 cleavage \\
\hline & & & &
\end{tabular}

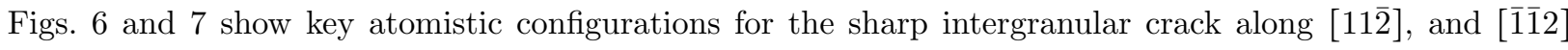
directions of twin boundary, respectively. The theoretical cleavage load both with and without hydrogen is $1.03 \mathrm{MPa} \sqrt{\mathrm{m}}$ since $\mathrm{H}$ binds weakly to this twin. For the case in which the crack is along $[11 \overline{2}]$ direction, the theoretical emission load is $1.01 \mathrm{MPa} \sqrt{\mathrm{m}}$, quite close but less than the theoretical cleavage load. In the H-free specimen, the crack starts to propagate at $1.20 \mathrm{MPa} \sqrt{\mathrm{m}}$, slightly higher than the emission/cleavage predictions. For the $\mathrm{H}$-charged case, with hydrogen atoms covering the crack faces, an initial $\mathrm{K}=0.80 \mathrm{MPa} \sqrt{\mathrm{m}}$ is applied to avoid closure of the crack. In this case, cleavage occurs at $1.20 \mathrm{MPa} \sqrt{\mathrm{m}}$, equal to the critical cleavage load for the $\mathrm{H}$-free case. This result is expected because the twin boundary does not absorb any $\mathrm{H}$ atom. In the case in which the crack is along the theoretically ductile direction [11̄2], the predicted emission load is $0.38 \mathrm{MPa} \sqrt{\mathrm{m}}$, whereas the simulations without $\mathrm{H}$ show emission at $0.624 \mathrm{MPa} \sqrt{\mathrm{m}}$. With $\mathrm{H}$, the emission load decreases slightly to $0.58 \mathrm{MPa} \sqrt{\mathrm{m}}$. Both with and without $\mathrm{H}$, the crack tip emits several partial dislocations. $\mathrm{H}$ thus does not change the ductile/brittle nature of the twin boundary, with most effects here associated with $\mathrm{H}$ absorbed on the pre-existing crack faces rather than with $\mathrm{H}$ segregated to the twin boundary itself.

Figs. 8 and 9 show key atomistic configurations for the intergranular crack along the $\mathrm{Ni} \Sigma 9(221)\langle 110\rangle$ boundary for the two directions of cracking, respectively. For the theoretical cleavage case, the predicted cleavage load decreases from $0.98 \mathrm{MPa} \sqrt{\mathrm{m}}$ in pure $\mathrm{Ni}$ to $0.97 \mathrm{MPa} \sqrt{\mathrm{m}}$ in the presence of $\mathrm{H}$, both less than the emission load of $1.50 \mathrm{MPa} \sqrt{\mathrm{m}}$. Thus, cleavage is predicted with and without $\mathrm{H}$. The simulations show that crack behavior is more complicated than a simple cleavage process. In pure $\mathrm{Ni}$, a tiny partial cleavage starts at $0.496 \mathrm{MPa} \sqrt{\mathrm{m}}$ but atoms at the crack tip rearrange and suppress continued cleavage. At a much higher load of 1.49 $\mathrm{MPa} \sqrt{\mathrm{m}}$, dislocations are emitted ahead of the crack tip, thus cleavage does not occur in this case - this theoretical cleavage case is in fact ductile. With $\mathrm{H}$, a tiny partial cleavage starts at $0.544 \mathrm{MPa} \sqrt{\mathrm{m}}$ followed by emission of dislocations at $1.10 \mathrm{MPa} \sqrt{\mathrm{m}}$ but eventually the crack faces cleave at $1.39 \mathrm{MPa} \sqrt{\mathrm{m}}$. This ductile-to- 
This is a pre-print of the following article: Tehranchi, A.; Curtin, W. A. Journal Of The Mechanics And Physics Of Solids 2017, 101, 150--165.. The formal publication is available at http://dx.doi.org/10.1016/j.jmps.2017.01.020

brittle transition for a case expected to be brittle without $\mathrm{H}$ may be explained if $\mathrm{H}$ reduces lattice trapping at the crack tip (see Discussion). For the theoretical emission case, the predicted emission load is $0.64 \mathrm{MPa} \sqrt{\mathrm{m}}$ and pure $\mathrm{Ni}$ shows emission at $0.72 \mathrm{MPa} \sqrt{\mathrm{m}}$, within the typical accuracy of the emission model. With $\mathrm{H}$, leading and twinning partials are emitted at the same time at a much higher load of $0.928 \mathrm{MPa} \sqrt{\mathrm{m}}$. H thus delays emission, but the emission load remains below the predicted cleavage load of $0.97 \mathrm{MPa} \sqrt{\mathrm{m}}$. The difference is small, however, indicating that $\mathrm{H}$ could push this boundary close to a transition from ductile-to-brittle, but no actual transition is observed in these simulations. The origin of the increase in the emission load is also not understood within the context of the emission theory, which does not account for any H-induced phenomena occurring right at the crack tip.

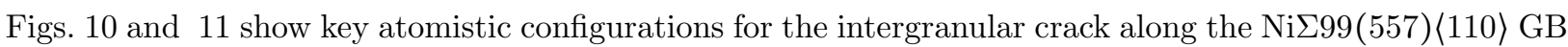

for the two directions of cracking, respectively. For the predicted cleavage case, the cleavage load decreases from $1.00 \mathrm{MPa} \sqrt{\mathrm{m}}$ in pure Ni to $0.92 \mathrm{MPa} \sqrt{\mathrm{m}}$ in the presence of H. Simulations in pure Ni show partial cleavage at $0.912 \mathrm{MPa} \sqrt{\mathrm{m}}$, followed by some atomic rearrangements at the crack tip, with cleavage finally occurring at 1.52 $\mathrm{MPa} \sqrt{\mathrm{m}}$. In the presence of $\mathrm{H}$, the final cleavage load is reduced to $1.072 \mathrm{MPa} \sqrt{\mathrm{m}}$, approaching the predicted values, with the $\mathrm{H}$ atoms preventing the local rearrangement at the crack tip. For the predicted emission case, simulations for pure $\mathrm{Ni}$ show emission at $0.624 \mathrm{MPa} \sqrt{\mathrm{m}}$ which is quite close to the predicted value of $0.62 \mathrm{MPa} \sqrt{\mathrm{m}}$. With $\mathrm{H}$, the emission load is reduced to $0.576 \mathrm{MPa} \sqrt{\mathrm{m}}$, slightly lower than the predicted value. As noted, distortions and energy of $\mathrm{H}$ near the crack tip are not included in the emission theory, and hence some deviations between simulation and emission theory can be expected. In any case, however, no ductile-to-brittle transition is observed due to the introduction of $\mathrm{H}$ into this GB.

Figs. 12 and 13 show key atomistic configurations for the intergranular crack along the $\mathrm{Ni} \Sigma 5(120)\langle 100\rangle$ for the two crack directions, respectively. Recall that for this boundary there are no slip planes containing the crack front so that any emission is on oblique planes, which requires higher loads and which is also outside the scope of the emission theory used here. Such three-dimensional behavior is beyond the scope of this work and so our simulations use short crack front lengths and are quasi-2d so as to suppress the oblique emission. Both crack directions are thus expected to be "cleavage" cases. The predicted cleavage load is $0.92 \mathrm{MPa} \sqrt{\mathrm{m}}$ in pure $\mathrm{Ni}$ and decreases to $0.75 \mathrm{MPa} \sqrt{\mathrm{m}}$ in the presence of $\mathrm{H}$. Simulations along [210] show partial cleavage at $0.75 \mathrm{MPa} \sqrt{\mathrm{m}}$ in pure Ni followed by rearrangement of the crack tip atoms around $0.87 \mathrm{MPa} \sqrt{\mathrm{m}}$. This rearrangement is a result of the tendency of the crack tip to emit oblique dislocation loops. Cleavage then proceeds at $0.91 \mathrm{MPa} \sqrt{\mathrm{m}}$, close to the predicted value. With $\mathrm{H}$, partial cleavage starts at $0.32 \mathrm{MPa} \sqrt{\mathrm{m}}$ followed by cleavage at $0.51 \mathrm{MPa} \sqrt{\mathrm{m}}$. This reduction in the cleavage load is strong evidence of the suppression of oblique dislocation emission by $\mathrm{H}$ atoms. For the crack along the opposite direction, partial cleavage occurs at $0.99 \mathrm{MPa} \sqrt{\mathrm{m}}$ in pure $\mathrm{Ni}$, followed by atomic rearranged at $1.04 \mathrm{MPa} \sqrt{\mathrm{m}}$ and cleavage at $1.2 \mathrm{MPa} \sqrt{\mathrm{m}}$. With $\mathrm{H}$, rearrangements occur at $0.90 \mathrm{MPa} \sqrt{\mathrm{m}}$ followed by cleavage at $1.14 \mathrm{MPa} \sqrt{\mathrm{m}}$, which is slightly lower than that of the pure Ni but larger than the predicted value. No ductile-to-brittle transition is observed due to the introduction of $\mathrm{H}$ into this $\mathrm{GB}$, and $\mathrm{H}$ generally facilitates cleavage, consistent with the trend of the theory. 
This is a pre-print of the following article: Tehranchi, A.; Curtin, W. A. Journal Of The Mechanics And Physics Of Solids 2017, 101, 150--165.. The formal publication is available at http://dx.doi.org/10.1016/j.jmps.2017.01.020

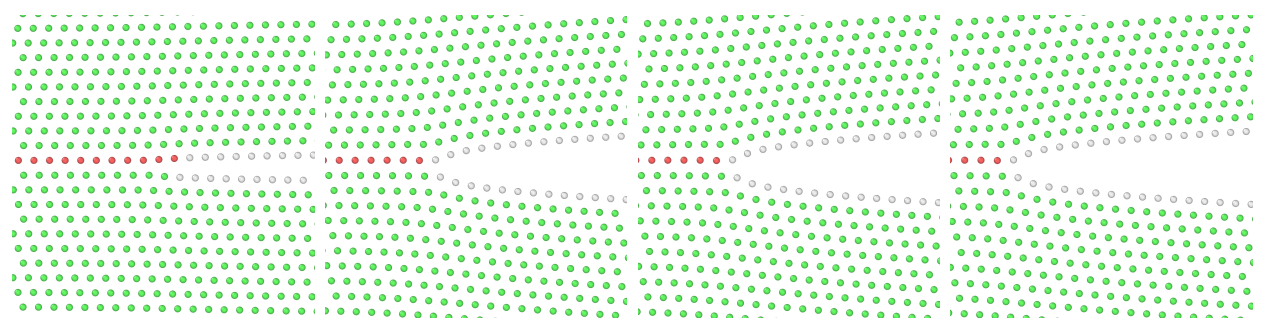
(a) $K_{I}^{\text {app. }}=0.24 \mathrm{MPa} \sqrt{m}$
(b) $K_{I}^{\mathrm{app} \cdot}=1.09 \mathrm{MPa} \sqrt{m}$
(c) $K_{I}^{\mathrm{app} \cdot}=1.18 \mathrm{MPa} \sqrt{m}$
(d) $K_{I}^{\mathrm{app}}=1.2 \mathrm{MPa} \sqrt{m}$

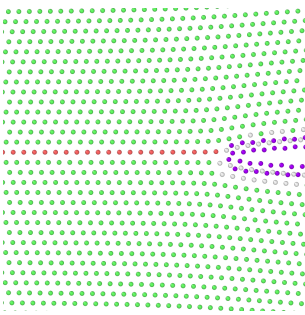

(e) $K_{I}^{\mathrm{app} \cdot}=0.80 \mathrm{MPa} \sqrt{m}$

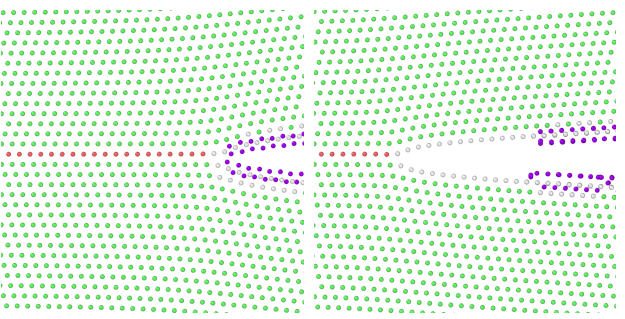

(f) $K_{I}^{\mathrm{app} \cdot}=1.18 \mathrm{MPa} \sqrt{m}$

(g) $K_{I}^{\mathrm{app} \cdot}=1.20 \mathrm{MPa} \sqrt{m}$

Figure 6: Sharp crack tip atomic structure at load levels where various events occur in the simulations of Ni $\Sigma 3(111)\langle 110\rangle$ along [11̄̄] direction. Atoms colored by Common Neighbor Analysis (Green: fcc Ni; Red: hcp Ni; White: Ni with less than 12 neighbors; Purple: H)

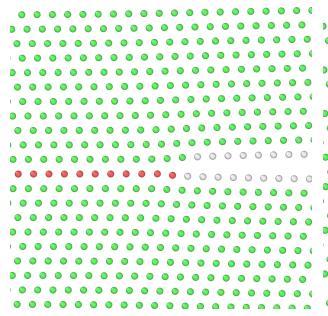

(a) $K_{I}^{\text {app. }}=0.24 \mathrm{MPa} \sqrt{m}$

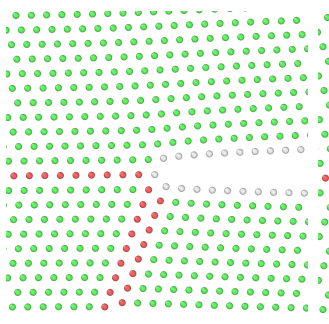

(b) $K_{I}^{\mathrm{app} \cdot}=0.624 \mathrm{MPa} \sqrt{m}$

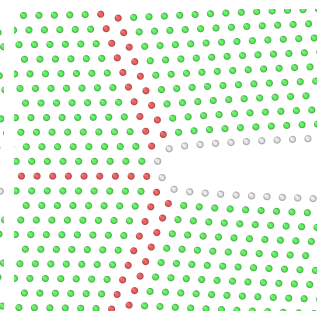

(c) $K_{I}^{\text {app. }}=0.896 \mathrm{MPa} \sqrt{m}$

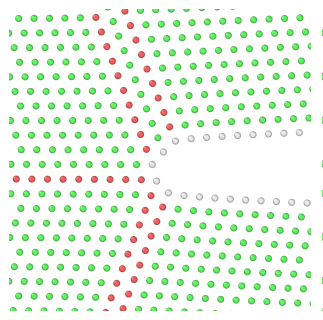

(d) $K_{I}^{\mathrm{app} .}=1.04 \mathrm{MPa} \sqrt{m}$

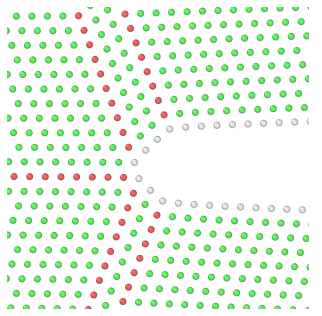

(e) $K_{I}^{\mathrm{app} .}=1.23 \mathrm{MPa} \sqrt{m}$

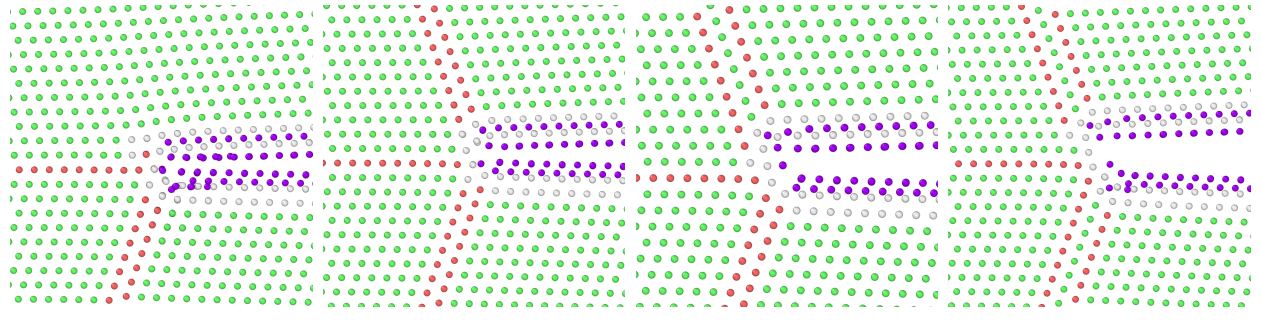
(f) $K_{I}^{\mathrm{app} .}=0.54 \mathrm{MPa} \sqrt{m}$
(g) $K_{I}^{\mathrm{app} \cdot}=0.58 \mathrm{MPa} \sqrt{m}$
(h) $K_{I}^{\text {app. }}=0.72 \mathrm{MPa} \sqrt{m}$
(i) $K_{I}^{\mathrm{app} .}=0.80 \mathrm{MPa} \sqrt{m}$

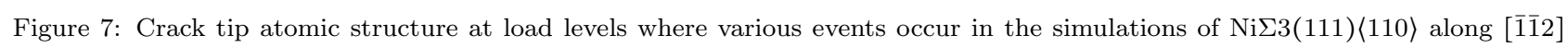
direction. Atoms colored by Common Neighbor Analysis (Green: fcc Ni; Red: hcp Ni; White: Ni with less than 12 neighbors; Purple: H) 
This is a pre-print of the following article: Tehranchi, A.; Curtin, W. A. Journal Of The Mechanics And Physics Of Solids 2017, 101, 150--165.. The formal publication is available at http://dx.doi.org/10.1016/j.jmps.2017.01.020

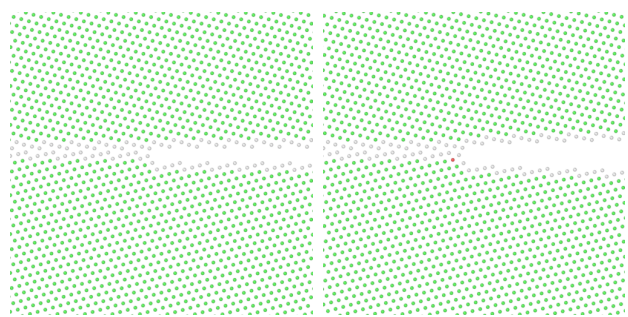

(a) $K_{I}^{\mathrm{app} \cdot}=0 \mathrm{MPa} \sqrt{m}$

(b) $K_{I}^{\mathrm{app}}=0.49 \mathrm{MPa} \sqrt{m}$

(c) $K_{I}^{\mathrm{app} \cdot}=1.28 \mathrm{MPa} \sqrt{m}$

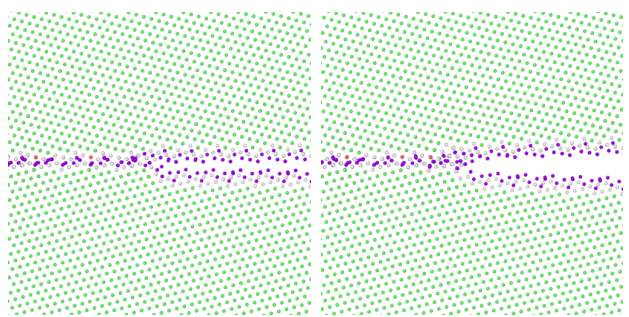

(e) $K_{I}^{\text {app. }}=0 \mathrm{MPa} \sqrt{m}$

(f) $K_{I}^{\text {app }}=0.54 \mathrm{MPa} \sqrt{m}$

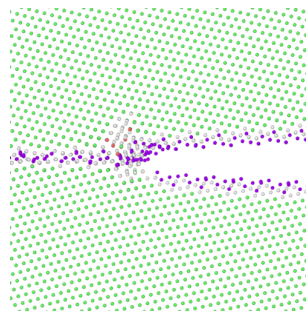

(g) $K_{I}^{\mathrm{app} \cdot}=1.10 \mathrm{MPa} \sqrt{m}$ (d) $K_{I}^{\mathrm{app} \cdot}=1.49 \mathrm{MPa} \sqrt{m}$
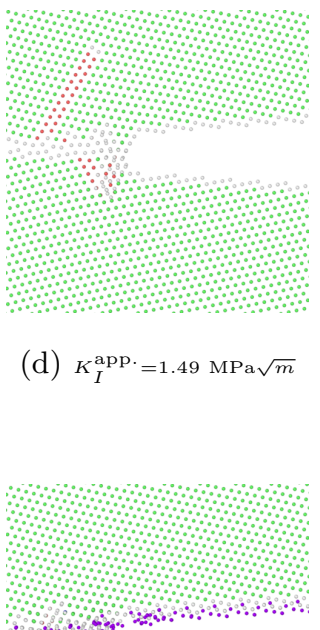
rent? m.m.

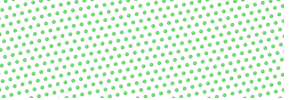

(h) $K_{I}^{\mathrm{app} \cdot}=1.39 \mathrm{MPa} \sqrt{m}$

Figure 8: Crack tip atomic structure at load levels where various events occur in the simulations of Ni $\Sigma 9(221)\langle 110\rangle$ along the theoretically cleavage favorable direction. Atoms colored by Common Neighbor Analysis (Green: fcc Ni; Red: hcp Ni; White: Ni with less than 12 neighbors; Purple: H)

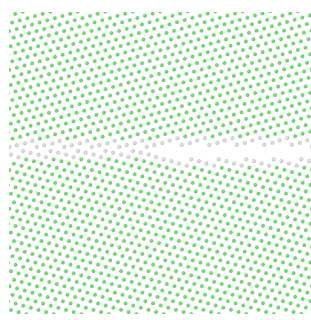

(a) $K_{I}^{\mathrm{app} \cdot}=0 \mathrm{MPa} \sqrt{m}$

(b) $K_{I}^{\text {app. }}=0.72 \mathrm{MPa} \sqrt{m}$

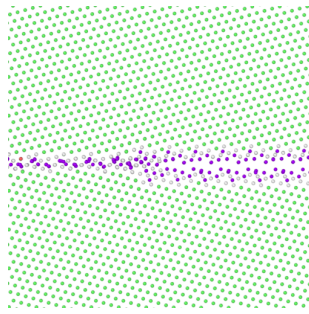

(e) $K_{I}^{\text {app. }}=0 \mathrm{MPa} \sqrt{m}$

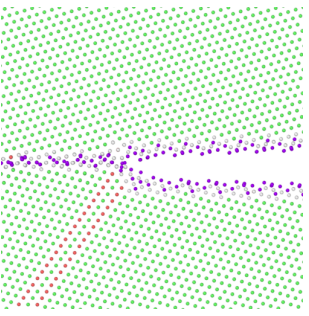

(f) $K_{I}^{\mathrm{app} \cdot}=0.93 \mathrm{MPa} \sqrt{m}$ (c) $K_{I}^{\text {app. }}=0.85 \mathrm{MPa} \sqrt{m}$

(d) $K_{I}^{\mathrm{app} .}=1.04 \mathrm{MPa} \sqrt{m}$
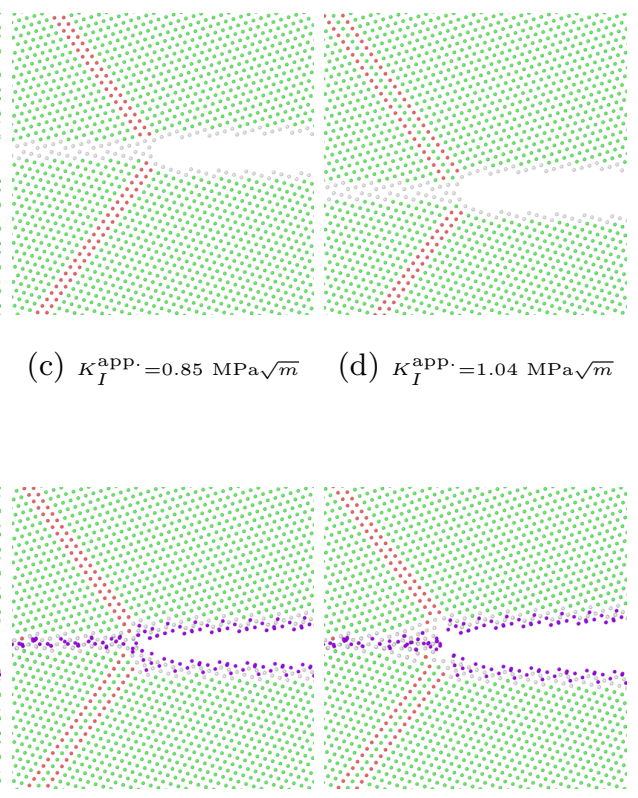

(g) $K_{I}^{\text {app. }}=0.99 \mathrm{MPa} \sqrt{m}$

Figure 9: Crack tip atomic structure at load levels where various events occur in the simulations of Ni $\Sigma 9(221)\langle 110\rangle$ along the theoretically emission favorable direction. Atoms colored by Common Neighbor Analysis (Green: fcc Ni; Red: hcp Ni; White: Ni with less than 12 neighbors; Purple: H) 
This is a pre-print of the following article: Tehranchi, A.; Curtin, W. A. Journal Of The Mechanics And Physics Of Solids 2017, 101, 150--165.. The formal publication is available at http://dx.doi.org/10.1016/j.jmps.2017.01.020

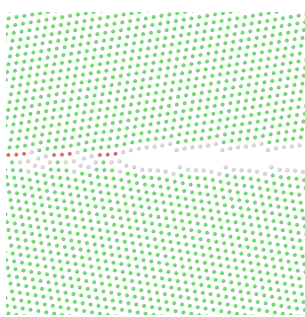

(a) $K_{I}^{\mathrm{app} .}=0 \mathrm{MPa} \sqrt{m}$

(b) $K_{I}^{\mathrm{app} \cdot}=0.91 \mathrm{MPa} \sqrt{m}$

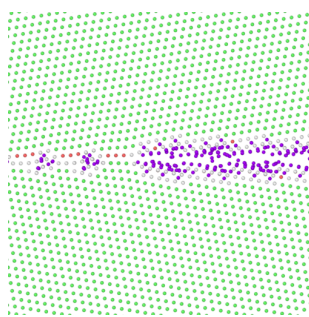

(e) $K_{I}^{\mathrm{app} \cdot}=0 \mathrm{MPa} \sqrt{m}$

(f) $K_{I}^{\mathrm{app} \cdot}=0.35 \mathrm{MPa} \sqrt{m}$ (c) $K_{I}^{\mathrm{app} .}=1.30 \mathrm{MPa} \sqrt{m}$

(d) $K_{I}^{\text {app. }}=1.52 \mathrm{MPa} \sqrt{m}$

(g) $K_{I}^{\mathrm{app} .}=0.61 \mathrm{MPa} \sqrt{m}$
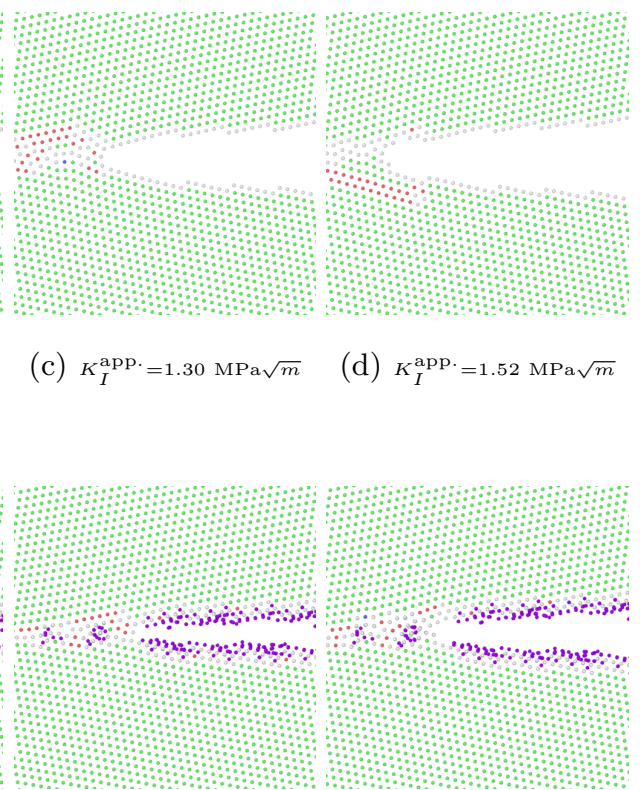

(h) $K_{I}^{\mathrm{app} \cdot}=1.07 \mathrm{MPa} \sqrt{m}$

Figure 10: Process of an intergranular crack propagation in Ni $\Sigma 99(557)\langle 110\rangle$ along the theoretically cleavage favorable direction. Atoms colored by Common Neighbor Analysis (Green: fcc Ni; Red: hcp Ni; White: Ni with less than 12 neighbors; Purple: H)

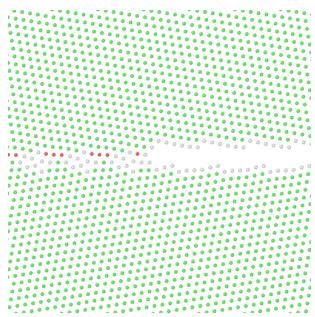

(a) $K_{I}^{\mathrm{app} \cdot}=0 \mathrm{MPa} \sqrt{m}$

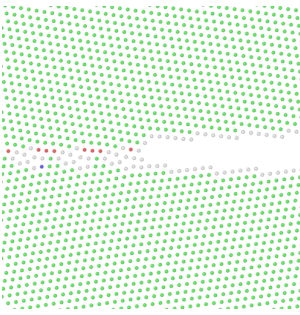

(b) $K_{I}^{\mathrm{app} \cdot}=0.45 \mathrm{MPa} \sqrt{m}$

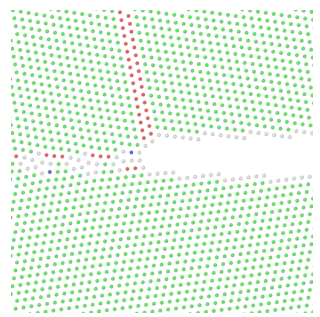

(c) $K_{I}^{\mathrm{app} \cdot}=0.63 \mathrm{MPa} \sqrt{m}$

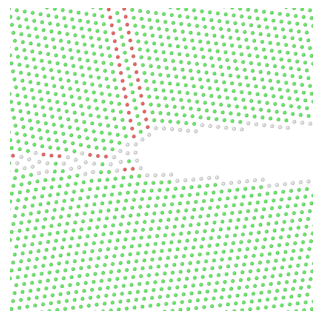

(d) $K_{I}^{\mathrm{app} \cdot}=1.04 \mathrm{MPa} \sqrt{m}$

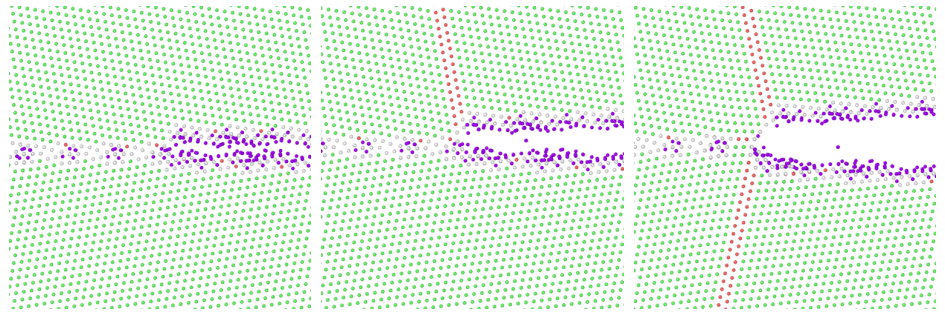
(e) $K_{I}^{\mathrm{app} \cdot}=0 \mathrm{MPa} \sqrt{m}$
(f) $K_{I}^{\text {app. }}=0.58 \mathrm{MPa} \sqrt{m}$
(g) $K_{I}^{\mathrm{app} \cdot}=1.28 \mathrm{MPa} \sqrt{m}$

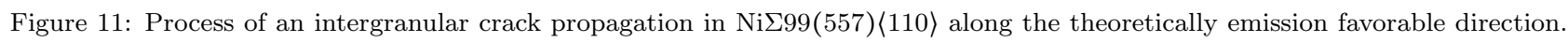
Atoms colored by Common Neighbor Analysis (Green: fcc Ni; Red: hcp Ni; White: Ni with less than 12 neighbors; Purple: H) 
This is a pre-print of the following article: Tehranchi, A.; Curtin, W. A. Journal Of The Mechanics And Physics Of Solids 2017, 101, 150--165.. The formal publication is available at http://dx.doi.org/10.1016/j.jmps.2017.01.020

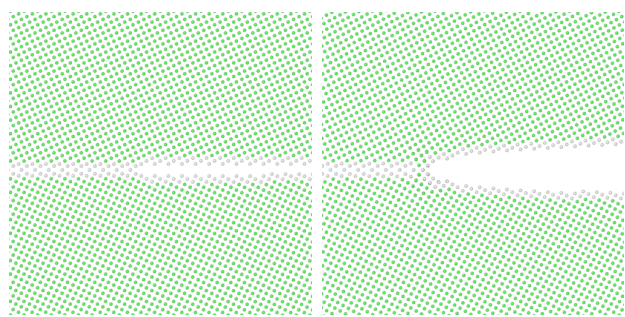

(a) $K_{I}^{\mathrm{app} \cdot}=0 \mathrm{MPa} \sqrt{m}$

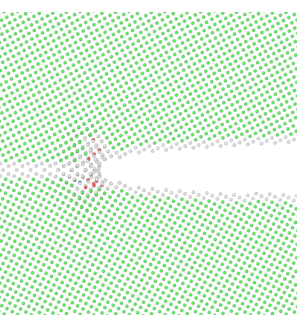

(c) $K_{I}^{\mathrm{app} \cdot}=0.87 \mathrm{MPa} \sqrt{m}$

(d) $K_{I}^{\mathrm{app} .}=0.91 \mathrm{MPa} \sqrt{m}$

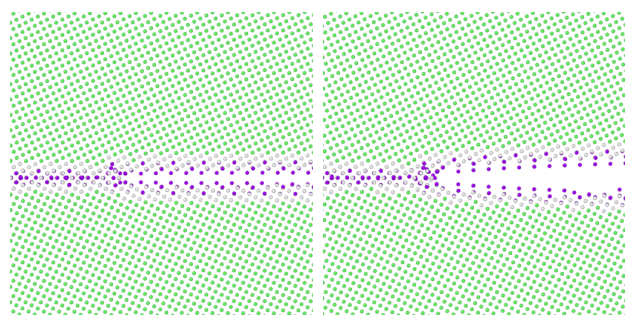

(e) $K_{I}^{\text {app. }}=0 \mathrm{MPa} \sqrt{m}$

(f) $K_{I}^{\mathrm{app} \cdot}=0.32 \mathrm{MPa} \sqrt{m}$

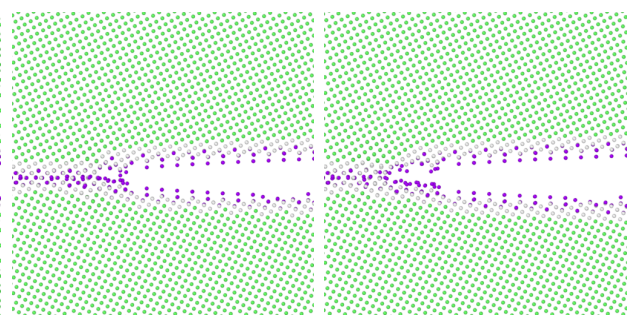

(g) $K_{I}^{\mathrm{app} \cdot}=0.51 \mathrm{MPa} \sqrt{m}$

(h) $K_{I}^{\mathrm{app} \cdot}=0.65 \mathrm{MPa} \sqrt{m}$

Figure 12: Process of an intergranular crack propagation in Ni $\Sigma 5(120)\langle 100\rangle$ along [2̄̄0] direction. Atoms colored by Common Neighbor Analysis (Green: fcc Ni; Red: hcp Ni; White: Ni with less than 12 neighbors; Purple: H)

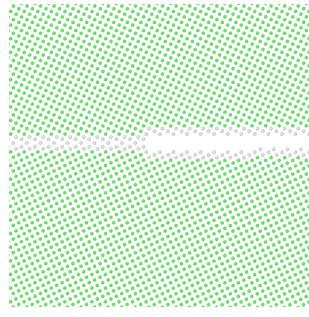

(a) $K_{I}^{\mathrm{app} \cdot}=0 \mathrm{MPa} \sqrt{m}$

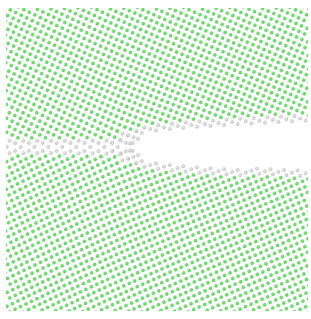

(b) $K_{I}^{\text {app. }}=0.99 \mathrm{MPa} \sqrt{m}$

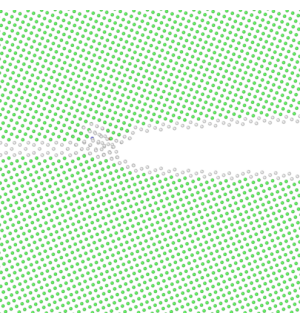

(c) $K_{I}^{\mathrm{app} .}=1.04 \mathrm{MPa} \sqrt{m}$

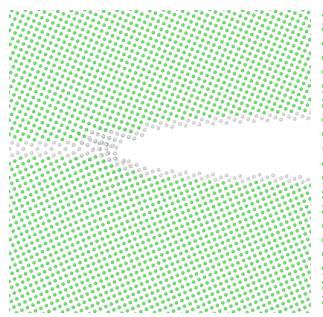

(d) $K_{I}^{\mathrm{app} \cdot}=1.20 \mathrm{MPa} \sqrt{m}$

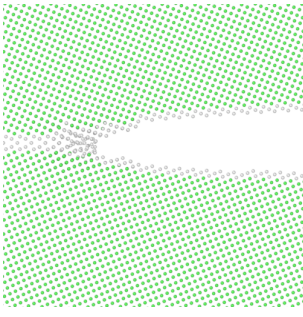

(e) $K_{I}^{\mathrm{app} \cdot}=1.31 \mathrm{MPa} \sqrt{m}$

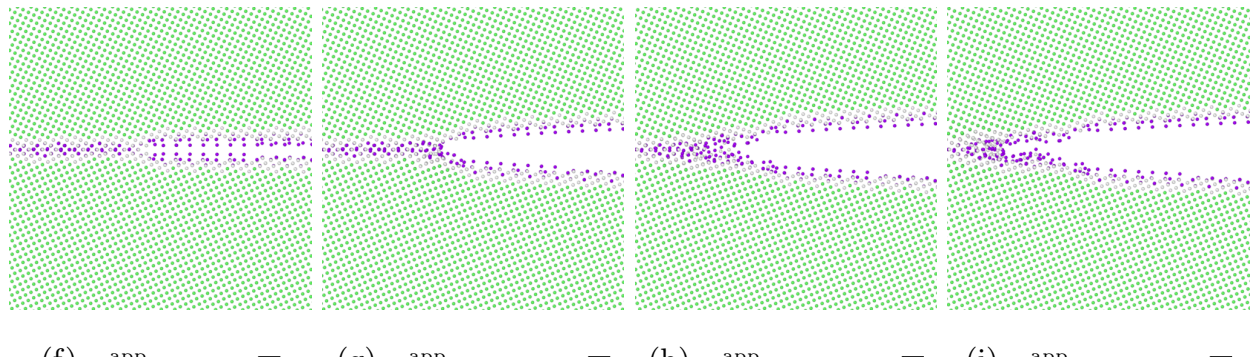

$\begin{array}{llll}\text { (f) } K_{I}^{\mathrm{app}}=0 \mathrm{MPa} \sqrt{m} & \text { (g) } K_{I}^{\mathrm{app} \cdot}=0.90 \mathrm{MPa} \sqrt{m} & \text { (h) } K_{I}^{\mathrm{app} .}=1.14 \mathrm{MPa} \sqrt{m} & \text { (i) } K_{I}^{\mathrm{app}}=1.20 \mathrm{MPa} \sqrt{m}\end{array}$

Figure 13: Process of an intergranular crack propagation in Ni $\Sigma 5(100)\langle 100\rangle$ along [210] direction. Atoms colored by Common Neighbor Analysis (Green: fcc Ni; Red: hcp Ni; White: Ni with less than 12 neighbors; Purple: H)

\section{Discussion}

The simulations show that no ductile-to-brittle transition is observed for the predicted ductile cracks in the presence of segregated hydrogen atoms along any of the GBs studied here. However, hydrogen does make cleavage easier for crack growth in the intrinsically brittle directions. In the particular case of Ni $\Sigma 9(221)\langle 110\rangle$,

the theoretically-brittle case is actually ductile, and the presence of $\mathrm{H}$ leads to the expected cleavage/brittle behavior. In the case of the twin boundary, which has no absorbed $\mathrm{H}$, the cleavage load remains unaltered. 
These observations indicate that hydrogen has limited embrittling effects due to a change in the crack tip behavior, but can help intergranular fracture to be more favorable in theoretically brittle cases. These results, except the case of $\mathrm{Ni} \Sigma 9(221)\langle 110\rangle$, are in qualitative agreement with the theoretical Rice and Griffith criteria. However in all cases, the exact values of the K-loads at which critical events occur are different between theory and simulation, and the nature of the different atomic scale mechanisms of crack growth vary. Below, we thus discuss the origins of these differences and further elucidate the effects of segregated hydrogen on these intergranular fracture processes.

One major effect that hinders easy cleavage of the crack faces, and that raises the fracture $K$ above the Griffith prediction, is crack trapping within the GB structure. Since the grain boundary has a complicated discrete structure at the atomistic level, the crack tip can be arrested within the higher-toughness regions of the GB. The crack may first extend through lower-toughness regions but ultimately encounters the locally-tougher regions. Griffith theory considers only the surface energy, which is an average over the various structural units in the GB. With trapping, a higher $K^{+}$is required for crack growth. If $K^{+}$is higher than the critical emission load, local plastic events can occur prior to, or in place of, cleavage. In pure Ni $\Sigma 9(221)\langle 110\rangle$, the final event in the predicted cleavage direction is actually emission ahead of the crack tip and thus no cleavage is observed. In this case, this final event occurs at a $K$ slightly higher than the theoretical Rice criterion and is a manifestation of trapping with $K^{+}>K_{I \mathrm{e}}$. Further evidence of strong trapping is seen for cracking in the [210] direction of the $\mathrm{Ni} \Sigma 5(120)\langle 100\rangle$ where, after a partial cleavage at a $K$ slightly higher than the theoretical cleavage $K$, the final cleavage occurs only after a further $50 \%$ increase in load. Finite temperatures allow thermal activation over trapping energy barriers, and so can alleviate trapping and facilitate cleavage at $K$ values lower than the value measured at $\mathrm{T}=0 \mathrm{~K}$.

The presence of $\mathrm{H}$ atoms could also help the crack tip overcome trapping. Specifically, the presence of $\mathrm{H}$ along the grain boundary occurs mainly in the open spaces of the boundary, presumably making the energy along the surface more uniform, and thus reducing trapping. In the predicted cleavage case for the $\mathrm{Ni \Sigma}(221)\langle 110\rangle$, in which the crack tip in pure $\mathrm{Ni}$ actually emits dislocations, adding $\mathrm{H}$ makes cleavage favorable by preventing local atomic rearrangements. This is the only case in which we observe any ductile-to-brittle transition. Per the discussion above, the embrittlement in this case presumably arises from reduction of trapping effects rather than reduction in the fracture energy. As stated earlier, temperature can reduce the trapping effect, thus even in this case we expect that embrittlement may not occur at finite-T. A similar effect reduces the critical K-load for cleavage in the predicted cleavage direction of $\mathrm{Ni} \Sigma 99(557)\langle 110\rangle$. The cleavage load remains higher than the Griffith prediction, but less than the theoretical emission load. For the crack along [210] of the Ni $\Sigma 5(120)\langle 100\rangle$, $\mathrm{H}$ atoms reduce the cleavage load by $8 \%$.

Another issue in relating simulations to theory is the occurrence of local distortions at the crack tip. These distortions could be an artifact of the interatomic potential or a consequence of complicated "plastic" activity at the crack tip. For an intergranular crack along [210] of the $\mathrm{Ni} \Sigma 5(120)\langle 100\rangle$, such plastic activity is the start of emission of oblique dislocation loops that, due to the short crack front length, interact and prevent larger-scale expansion of the dislocation loops. This activity hinders the crack from propagating until the load has been increased by an additional $15 \%$.

$\mathrm{H}$ atoms could also have both increasing and decreasing effects on the emission load. A high $\mathrm{H}$ concentration along the inclined slip planes in the grains will increase $\gamma_{\text {usf }}$ and hence increase the emission load [21]; this is not present in our simulations because $\mathrm{H}$ is only present in the grain boundaries. Emission in mode I loading is 
accompanied by creation of a surface step, and the energy cost for creation of this surface step is proportional to the surface energy of the metal; this effect is not included in the Rice theory. Since H atoms saturate the crack faces in most cases, the surface energy could be reduced and the critical $K$ for emission also reduced, but this depends on $\mathrm{H}$ rearrangements as the step is being created. Here, for the predicted emission directions of $\mathrm{Ni} \Sigma 3(111)\langle 110\rangle$ and $\mathrm{Ni} \Sigma 99(557)\langle 110\rangle$, the presence of $\mathrm{H}$ atoms along the GB and on the crack faces indeed reduces the critical $K$ for emission. In the case of the $\mathrm{Ni} \Sigma 3(111)\langle 110\rangle \mathrm{GB}$, where $\mathrm{H}$ atoms are present only on the crack faces, the reduction in the emission load is due to this effect. In the case of the $\mathrm{Ni} \Sigma 9(221)\langle 110\rangle$ $\mathrm{GB}$, adding $\mathrm{H}$ atoms does not decrease the emission load. However, the crack tip emitted both leading and twinning partials at the same $K$. Hydrogen-enhanced emission of dislocations from crack tips is reported in the literature, for example see [36].

All simulations and theoretical calculations presented in this work are done for $\mathrm{T}=0 K$, and finite- $\mathrm{T}$ thermal activation might be envisioned to alter the results. However, we believe the $\mathrm{T}=0 K$ results are reasonable for understanding most finite-T conditions. The Griffith theory is a thermodynamic criterion and so the effects of temperature are due only to temperature-dependent changes in surface/interface energies and elastic moduli. These quantities both typically decrease with increasing temperature. Thus, the $K$ for cleavage should reduce with increasing temperature. The Rice criterion is an instability condition, and so there is an energy barrier that can be overcome at lower loads at finite T. However, the energy barriers are typically large [37, 38, and so the decrease in applied $K$ is relatively small at moderate temperatures $(\mathrm{T}=300 \mathrm{~K})$. The main effects of temperature on the Rice condition are thus due to changes in $\gamma_{\text {usf }}$ and elastic moduli. The decrease in $K$ for emission is thus expected to change similarly to the $K$ for cleavage. Thus, the temperature-dependent competition between cleavage and emission will not change dramatically and predictions at $\mathrm{T}=0 K$ are thus expected to be representative of results at finite $\mathrm{T}$, in general. The only exception to this discussion involves lattice trapping, where we noted earlier that finite temperatures will reduce trapping and allow cleavage to occur closer to the thermodynamic Griffith limit.

In summary, the presence of $\mathrm{H}$ creates no ductile-to-brittle transition for grain boundary cracks that are predicted to emit dislocations in the absence of $\mathrm{H}$, at levels of $\mathrm{H}$ corresponding to equilibrium segregation at bulk $\mathrm{H}$ concentrations where embrittlement is typically observed in $\mathrm{Ni}$. The simulation results are in qualitative agreement with the Griffith and Rice theories, although quantitative differences are notable. Reasons for the differences have been discussed. Generally, H makes cleavage possible and easier in the predicted brittle cases, but does not cause the predicted emission cases to become brittle. For a finite length crack nucleated along a grain boundary that is saturated with an equilibrium distribution of $\mathrm{H}$ atoms, the crack is predicted to propagate only along the brittle direction. This propagation either needs higher $\mathrm{K}$ or is not possible in the $\mathrm{H}-$ free case. The crack tip in the ductile direction will not extend by cleavage and, as the crack grows in the brittle direction and $K$ increases, the crack would emit dislocations in the ductile direction. We find no evidence that $\mathrm{H}$ changes the ductile behavior in theoretically ductile cases, and in fact observe cases where $\mathrm{H}$ facilitates emission. While no study can be exhaustive, our results lead us to conclude that hydrogen embrittlement observed in polycrystalline $\mathrm{Ni}$ is not likely associated with equilibrium-segregated $\mathrm{H}$ along grain boundaries. Embrittlement is thus presumably attributed to processes that involve $\mathrm{H}$ transport (through the bulk or along the boundaries), such as the mechanism proposed by Song and Curtin [19, 20. Other effects of $\mathrm{H}$ on dislocations may affect the plastic evolution, but these effects do not directly point toward intergranular cleavage-like failure. Our results for the twin boundary reveal that this boundary does not absorb $\mathrm{H}$ at all. Hence, as shown experimentally 
by [39, increasing the fraction of twin boundaries by grain boundary engineering processes is one strategy for reducing embrittlement in Ni. Nonetheless, the mechanisms of embrittlement in fcc metals thus remain open to further theoretical investigation.

\section{Acknowledgments}

The authors gratefully acknowledge support of this work from the Swiss National Foundation through a grant for the project entitled "Predictive Mechanisms of Hydrogen Embrittlement" (project \# 200021-149207).

\section{References}

[1] R. N. Iyer, H. W. Pickering, Mechanism and kinetics of electrochemical hydrogen entry and degradation of metallic systems, Annual Review of Materials Science 20 (1) (1990) 299-338.

[2] S. M. Myers, M. Baskes, H. Birnbaum, J. W. Corbett, G. DeLeo, S. Estreicher, E. E. Haller, P. Jena, N. M. Johnson, R. Kirchheim, et al., Hydrogen interactions with defects in crystalline solids, Reviews of Modern Physics 64 (2) (1992) 559.

[3] H. Vehoff, Hydrogen related material problems, in: Hydrogen in metals III, Springer, 1997, pp. 215-278.

[4] S. Lynch, Mechanisms of hydrogen assisted cracking-a review, Hydrogen Effects on Material Behaviour and Corrosion Deformation Interactions (2003) 449-466.

[5] R. P. Gangloff, Hydrogen assisted cracking of high strength alloys, Tech. rep., DTIC Document (2003).

[6] P. Sofronis, I. Robertson, Transmission electron microscopy observations and micromechanical/continuum models for the effect of hydrogen on the mechanical behaviour of metals, Philosophical Magazine A 82 (1718) (2002) 3405-3413.

[7] P. Ferreira, I. Robertson, H. Birnbaum, Hydrogen effects on the interaction between dislocations, Acta materialia 46 (5) (1998) 1749-1757.

[8] D. P. Abraham, C. J. Altstetter, Hydrogen-enhanced localization of plasticity in an austenitic stainless steel, Metallurgical and Materials transactions A 26 (11) (1995) 2859-2871.

[9] Y. Murakami, The effect of hydrogen on fatigue properties of metals used for fuel cell system, in: Advances in Fracture Research, Springer, 2006, pp. 167-195.

[10] A. Barnoush, H. Vehoff, In situ electrochemical nanoindentation: A technique for local examination of hydrogen embrittlement, Corrosion Science 50 (1) (2008) 259-267.

[11] V. Gaspard, G. Kermouche, D. Delafosse, A. Barnoush, Hydrogen effect on dislocation nucleation in a ferritic alloy fe- $-15 \mathrm{cr}$ as observed per nanoindentation, Materials Science and Engineering: A 604 (2014) $86-91$.

[12] M. Wen, Z. Li, A. Barnoush, Atomistic study of hydrogen effect on dislocation nucleation at crack tip, Advanced Engineering Materials 15 (11) (2013) 1146-1151. 
This is a pre-print of the following article: Tehranchi, A.; Curtin, W. A. Journal Of The Mechanics And Physics Of Solids 2017, 101, 150--165.. The formal publication is available at http://dx.doi.org/10.1016/j.jmps.2017.01.020

[13] S. Taketomi, R. Matsumoto, N. Miyazaki, Atomistic study of the effect of hydrogen on dislocation emission from a mode ii crack tip in alpha iron, International Journal of Mechanical Sciences 52 (2) (2010) 334-338.

[14] R. Matsumoto, S. Taketomi, S. Matsumoto, N. Miyazaki, Atomistic simulations of hydrogen embrittlement, international journal of hydrogen energy 34 (23) (2009) 9576-9584.

[15] Z. Hu, S. Fukuyama, K. Yokogawa, S. Okamoto, Hydrogen embrittlement of a single crystal of iron on a nanometre scale at a crack tip by molecular dynamics, Modelling and Simulation in Materials Science and Engineering 7 (4) (1999) 541.

[16] X. Xu, M. Wen, Z. Hu, S. Fukuyama, K. Yokogawa, Atomistic process on hydrogen embrittlement of a single crystal of nickel by the embedded atom method, Computational materials science 23 (1) (2002) $131-138$.

[17] J. von Pezold, L. Lymperakis, J. Neugebeauer, Hydrogen-enhanced local plasticity at dilute bulk h concentrations: The role of $\mathrm{h}-\mathrm{h}$ interactions and the formation of local hydrides, Acta Materialia 59 (8) (2011) $2969-2980$.

[18] M. Q. Chandler, M. Horstemeyer, M. Baskes, P. Gullett, G. Wagner, B. Jelinek, Hydrogen effects on nanovoid nucleation in face-centered cubic single-crystals, Acta Materialia 56 (1) (2008) 95-104.

[19] J. Song, W. Curtin, A nanoscale mechanism of hydrogen embrittlement in metals, Acta Materialia 59 (4) (2011) $1557-1569$.

[20] J. Song, W. Curtin, Atomic mechanism and prediction of hydrogen embrittlement in iron, Nature materials 12 (2) (2013) 145-151.

[21] J. Song, M. Soare, W. Curtin, Testing continuum concepts for hydrogen embrittlement in metals using atomistics, Modelling and Simulation in Materials Science and Engineering 18 (4) (2010) 045003.

[22] Z. Wu, W. Curtin, Brittle and ductile crack-tip behavior in magnesium, Acta Materialia 88 (2015) 1-12.

[23] J. R. Rice, Dislocation nucleation from a crack tip: an analysis based on the peierls concept, Journal of the Mechanics and Physics of Solids 40 (2) (1992) 239-271.

[24] J.-S. Wang, P. Anderson, Fracture behavior of embrittled fcc metal bicrystals, Acta metallurgica et materialia 39 (5) (1991) 779-792.

[25] J.-S. Wang, S. D. Mesarovic, Directional dependence of corrosion fatigue of iron-silicon bicrystals, Acta metallurgica et materialia 43 (10) (1995) 3837-3849.

[26] X. Zhou, D. Marchand, D. L. McDowell, T. Zhu, J. Song, Chemomechanical origin of hydrogen trapping at grain boundaries in fcc metals, Physical review letters 116 (7) (2016) 075502.

[27] J. E. Angelo, N. R. Moody, M. I. Baskes, Trapping of hydrogen to lattice defects in nickel, Modelling and Simulation in Materials Science and Engineering 3 (3) (1995) 289.

[28] M. Baskes, X. Sha, J. Angelo, N. Moody, Trapping of hydrogen to lattice defects in nickel, Modelling and Simulation in Materials Science and Engineering 5 (6) (1997) 651. 
${ }_{415}[29]$ A. Alvaro, I. T. Jensen, N. Kheradmand, O. Løvvik, V. Olden, Hydrogen embrittlement in nickel, visited by first principles modeling, cohesive zone simulation and nanomechanical testing, international journal of hydrogen energy 40 (47) (2015) 16892-16900.

[30] D. Di Stefano, M. Mrovec, C. Elsässer, First-principles investigation of hydrogen trapping and diffusion at grain boundaries in nickel, Acta Materialia 98 (2015) 306-312.

[31] D. Jiang, E. A. Carter, First principles assessment of ideal fracture energies of materials with mobile impurities: implications for hydrogen embrittlement of metals, Acta materialia 52 (16) (2004) 4801-4807.

[32] S. Plimpton, Fast parallel algorithms for short-range molecular dynamics, Journal of computational physics 117 (1) (1995) 1-19.

[33] A. Stukowski, Visualization and analysis of atomistic simulation data with ovito-the open visualization tool, Modelling and Simulation in Materials Science and Engineering 18 (1) (2009) 015012.

[34] J. R. Rice, Hydrogen and interfacial cohesion, Metallurgical Society of AIME (1976) 455-466.

[35] M. Hestenes, E. Stiefel, Methods of conjugate gradients for solving linear systems, Journal of Research of the National Bureau of Standards 49 (6) (1952) 409.

[36] S. Lynch, A fractographic study of hydrogen-assisted cracking and liquid-metal embrittlement in nickel, Journal of Materials science 21 (2) (1986) 692-704.

[37] V. Yamakov, D. Warner, R. Zamora, E. Saether, W. Curtin, E. Glaessgen, Investigation of crack tip dislocation emission in aluminum using multiscale molecular dynamics simulation and continuum modeling, Journal of the Mechanics and Physics of Solids 65 (2014) 35-53.

[38] D. Warner, W. Curtin, S. Qu, Rate dependence of crack-tip processes predicts twinning trends in fcc metals, Nature materials 6 (11) (2007) 876-881.

[39] S. Bechtle, M. Kumar, B. P. Somerday, M. E. Launey, R. O. Ritchie, Grain-boundary engineering markedly reduces susceptibility to intergranular hydrogen embrittlement in metallic materials, Acta Materialia 57 (14) (2009) 4148-4157. 